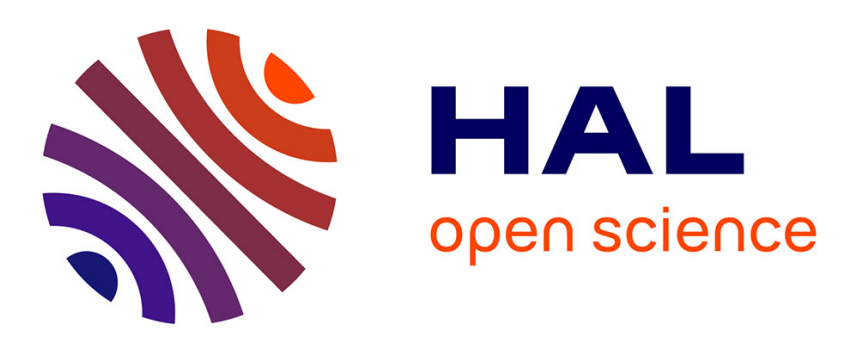

\title{
Lattice-Boltzmann Large-Eddy Simulation of pollutant dispersion in street canyons including tree planting effects
}

\author{
Lucie Merlier, Jérôme Jacob, Pierre Sagaut
}

\section{- To cite this version:}

Lucie Merlier, Jérôme Jacob, Pierre Sagaut. Lattice-Boltzmann Large-Eddy Simulation of pollutant dispersion in street canyons including tree planting effects. Atmospheric Environment, 2018, 195, pp.89-103. 10.1016/j.atmosenv.2018.09.040 . hal-02114676

\section{HAL Id: hal-02114676 \\ https://hal-amu.archives-ouvertes.fr/hal-02114676}

Submitted on 29 Apr 2019

HAL is a multi-disciplinary open access archive for the deposit and dissemination of scientific research documents, whether they are published or not. The documents may come from teaching and research institutions in France or abroad, or from public or private research centers.
L'archive ouverte pluridisciplinaire HAL, est destinée au dépôt et à la diffusion de documents scientifiques de niveau recherche, publiés ou non, émanant des établissements d'enseignement et de recherche français ou étrangers, des laboratoires publics ou privés. 


\section{Accepted Manuscript}

Lattice-Boltzmann Large-Eddy Simulation of pollutant dispersion in street canyons including tree planting effects

Lucie Merlier, Jérôme Jacob, Pierre Sagaut

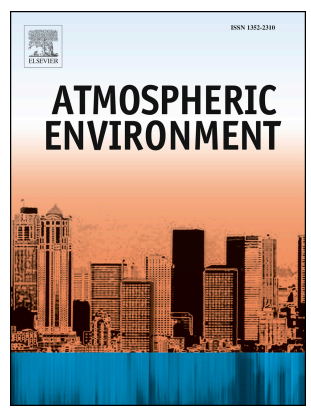

PII:

S1352-2310(18)30640-X

DOI:

10.1016/j.atmosenv.2018.09.040

Reference: $\quad$ AEA 16273

To appear in: Atmospheric Environment

Received Date: 10 April 2018

Revised Date: 18 September 2018

Accepted Date: 21 September 2018

Please cite this article as: Merlier, L., Jacob, Jéô., Sagaut, P., Lattice-Boltzmann Large-Eddy Simulation of pollutant dispersion in street canyons including tree planting effects, Atmospheric Environment (2018), doi: https://doi.org/10.1016/j.atmosenv.2018.09.040.

This is a PDF file of an unedited manuscript that has been accepted for publication. As a service to our customers we are providing this early version of the manuscript. The manuscript will undergo copyediting, typesetting, and review of the resulting proof before it is published in its final form. Please note that during the production process errors may be discovered which could affect the content, and all legal disclaimers that apply to the journal pertain. 


\title{
Lattice-Boltzmann Large-Eddy Simulation of pollutant dispersion in street canyons including tree planting effects
}

\author{
Lucie Merlier $^{\mathrm{a}}$, Jérôme Jacob ${ }^{\mathrm{a}}$, Pierre Sagaut ${ }^{\mathrm{a}}$ \\ ${ }^{a}$ Aix Marseille Univ, CNRS, Centrale Marseille, M2P2 UMR 7340, Marseille, France
}

\section{Abstract}

This study assesses the performance of a large eddy simulation (LES) based on the lattice Boltzmann method (LBM) in predicting near field dispersion in street canyons with tree planting. Based on a benchmark test case benefiting from wind tunnel measurements (CODASC), this study qualitatively and quantitatively discusses the prediction of traffic-induced pollutant concentration with respect to several reference studies. It also analyses the physics of the flow and concentration fields. Although the problem might seem rather simple, the flow is highlighted to be strongly three dimensional and transient. These properties enhance pollutant dispersion in the empty street canyon but air flow velocity and turbulence intensity tend to decrease in tree crowns. This effect of trees increases both mean and peak concentration levels at pedestrian level, which may be problematic in cities with dense traffic. These results show that LBM-LES is particularly well suited to study dispersion problems towards the development of more breathable cities. Keywords: Computational Fluid Dynamics, Lattice Boltzmann Method,

Email address: jerome.jacob@univ-amu.fr (Jérôme Jacob) 
Large Eddy Simulation, Urban pollutant dispersion, 3D Street canyon, Tree crowns.

\section{Introduction}

In the current context of environmental stress, near-field pollutant dispersion issues due to anthropogenic activities are of major concern. According to the World Health Organization's urban ambient air pollution database, more than $80 \%$ of people living in cities, for which monitored data are available, face pollution concentration levels that exceed recommendations. This is especially the case in low-income regions (World Heath Organization, 2016). A large part of pollutant emission in urban areas is due to transport. Vehicles emit 30\% of particle material in European cities. This ratio reaches $50 \%$ in the OECD countries, especially because of diesel use. This pollution substantially increases risks of diseases and prematured death rates in cities (World Heath Organization, 2018). Hence, while greenery is currently promoted as a solution to improve urban environmental quality including urban micro-climates and air quality thanks to filtering and deposition on plant foils, the inverse effect of trees on pollutant dispersion in dense cities with street canyons may be problematic (Gromke and Ruck, 2007, 2009; Janhäll, 2015; Abhijith and Gokhale, 2015; Abhijith et al., 2017; Jeanjean et al., 2017; Santiago et al., 2017; Xue and Li, 2017): tree crowns also tend to curb airflow and reduce the natural ventilation potential of streets, thus increasing pedestrian exposure to high levels of pollutant concentration. Wise urban planning choices should consequently be made to improve the breathability of urban areas in a context of climate change. However, in urban environments that 
include sharp-edged buildings and trees, air flows and pollutant dispersion processes are complex (Britter and Hanna, 2003; Ahmad et al., 2005; Lateb et al., 2016), making predictions and thus decisions more difficult.

Hence, for several decades now, different approaches to study urban flow and dispersion issues were developed. Thanks to the recent progress in computational capabilities, the use of detailed numerical approaches - typically computational fluid dynamics (CFD) - has increased, improving the accuracy of predictions. Capable of providing whole flow field data, this investigation technique advantageously completes experimental approaches and systematic field measurements. Although requiring an appropriate use and implementation, CFD is especially beneficial to highlight basic aerodynamic mechanisms underlying dispersion and to study virtual scenarios (Vardoulakis et al., 2003; Moonen et al., 2012; Tominaga and Stathopoulos, 2013; Blocken, 2015; Lateb et al., 2016). In urban physics, most studies rely on steady statistically averaged methods (Reynolds Averaged Navier-Stokes - RANS), because of the smaller computational costs involved. However, the accuracy of usual steady RANS approaches for studying dispersion in built environments is often found rough because of their inherent limitation in solving transient processes and turbulent transfers, which are important for dispersion (Tominaga and Stathopoulos, 2011; Salim and Ong, 2013; Tominaga and Stathopoulos, 2016). The use of time dependent approaches that resolve large scales of turbulence - typically large eddy simulation (LES) - appears therefore effective, but this also raises additional modeling challenges compared to RANS (Blocken, 2014, 2015). Especially, suitable boundary conditions should be specified (Tabor and Baba-Ahmadi, 2010), and the efficiency of the solver 
used is critical due to the increased computational costs LES involves in comparison to RANS.

The development and use of effective simulation approaches such as Lattice Boltzmann Method (LBM (Chen and Doolen, 1998; Succi, 2001; Shan et al., 2006; Guo and Shu, 2013; Krüger et al., 2017))-based LES approaches appear thus promising for urban applications. Indeed, thanks to its local and explicit formulation compared to Navier-Stokes-based approaches, this method is inherently parallel and very efficient to simulate low Mach separated flows. Regarding urban applications, first uses of this method addressed the simulation and visualization of contaminant dispersion using GPU (Graphics Processing Unit) for civil security (Fan et al., 2004; Qiu et al., 2004). Ten years later, this approach is receiving more and more interest because of its efficiency, although being still an emergent method. Contemporary studies especially address its accuracy and computational performance when implemented on GPUs (Obrecht et al., 2015; King et al., 2017), take advantage of this massively parallelizable method to discuss the link between urban morphology and pedestrian comfort (Ahmad et al., 2017; Jacob and Sagaut, 2018), or to quantify uncertainties or assimilate data for pollutant dispersion (Margheri and Sagaut, 2016; Mons et al., 2017).

To examine further the applicability and performance of LBM LES for urban issues, the present paper discusses qualitatively and quantitatively the accuracy of such an approach to address dispersion problems in the urban canopy layer. More specifically, the present study discusses results of LBM LES performed using ProLB (CS, 2018; M2P2, 2018) with respect to a benchmark test case: the COncentration DAta of Street Canyons - CODASC (KIT, 
2017). The CODASC focuses on pollutant dispersion in a street canyon with traffic-like pollutant emissions for different configurations of avenue-like tree planting. This configuration is used to analyze the physical processes that underlay dispersion in the urban canopy layer, as made possible by high fidelity modeling approaches.

The present paper is organized as follows. First, Sec. 2 presents the key features of the LBM LES approach used for this study. Second, Sec. 3 is dedicated to the CODASC benchmark in terms of experiment (Sec. 3.1) and related numerical studies (Sec. 3.2). Then Sec. 4 discusses the modeling we developed using ProLB (Sec. 4.1). It also presents the grid sensitivity analysis results (Sec. 4.2) and qualitatively and quantitatively discusses simulation results with respect to experimental data (Sec. 4.3). On this basis, Sec. 5 analyses the physics of the flow and the associated turbulent dispersion processes. Finally, Sec. 6 synthesizes the main results of this study and gives outlooks.

\section{The hybrid LBM LES approach}

General approach. The Boltzmann equation is a statistical equation, which describes the evolution of the distribution function $f$ of a particle of mass $m$ and speed $\xi$ undergoing an external force $\mathbf{F}$ in a fluid:

$$
\frac{\partial f}{\partial t}+\xi \cdot \frac{\partial f}{\partial \mathbf{x}}+\frac{\mathbf{F}}{m} \cdot \frac{\partial f}{\partial \xi}=\Omega(f)
$$

where $\Omega(f)$ is a collision operator standing for particle interactions during shocks. 
Based on a mesoscopic description, the LBM aims at simulating the fluid behavior by resolving a discretized version of the Boltzmann equation in phase space, using (CS, 2016):

1. a discrete velocity model $c_{\alpha, \alpha=0 \ldots Q-1}$ in a space of dimension $D$. A D3Q19 scheme is typically used for 3D problems;

2. a collision model towards relaxation. The simplest model is the single relaxation time Bhatnagar-Gross-Krook (BGK) model:

$$
\Omega=-\frac{1}{\tau}\left(f-f^{e q}\right)
$$

with: $\left\{\begin{array}{l}\tau: \text { the relaxation time, } \\ f^{e q}: \text { the equilibrium function; }\end{array}\right.$

3. and an equilibrium function model. This function generally corresponds to the development of the Maxwell-Boltzmann distribution function.

After projection and integration of Eq. 1, and neglecting at first external forces, the LBM BGK formulation reads:

$$
f_{\alpha}\left(\mathbf{x}+\mathbf{c}_{\alpha} \Delta t, t+\Delta t\right)-f_{\alpha}(\mathbf{x}, t)=-\frac{\Delta t}{\tau}\left(f_{\alpha}(\mathbf{x}, t)-f_{\alpha}^{e q}(\mathbf{x}, t)\right)
$$

The left hand side of Eq. 3 corresponds to the stream phase and the right hand side of Eq. 3 corresponds to the collision phase. The development of the equilibrium function to the second order is given by :

$$
f_{\alpha}^{e q}(\mathbf{x}, t)=\rho \omega_{\alpha}\left(1+\frac{c_{\alpha i} u_{i}}{c_{s}^{2}}+\frac{1}{2 c_{s}^{4}} Q_{\alpha i j} u_{i} u_{j}\right)
$$


${ }_{111}$ with: $\left\{\begin{array}{l}\omega_{\alpha} \text { and } c_{s} \text { : weight and sound velocity constants depending on the lattice used, } \\ Q_{\alpha i j}=c_{\alpha i} c_{\alpha j}-c_{s}^{2} \delta_{i j} .\end{array}\right.$

From LBM, usual macroscopic quantities such as the fluid density $\rho$ and flow momentum $\rho \mathbf{u}$ can be recovered as follows:

$$
\rho=\sum_{\alpha} f_{\alpha} ; \quad \rho \mathbf{u}=\sum_{\alpha} f_{\alpha} \mathbf{c}_{\alpha}
$$

Also, with the the BGK collision operator, the kinematic viscosity $\nu$ is related to the relaxation time $\tau$, following:

$$
\nu=c_{s}^{2}\left(\tau-\frac{\Delta t}{2}\right)
$$

This general LBM framework model allows to recover the Navier-Stokes equation to the second order and is the basis of the CFD solver ProLB. Nevertheless, in order to enhance the stability of computation while keeping the simplicity and accuracy of the scheme, a third-order expansion of the equilibrium function was used in the present study, along with a hybrid Recursive Reconstruction procedure for the non-equilibrium part of the distribution function $f_{\alpha}^{n e q}=f_{\alpha}-f_{\alpha}^{e q}$ (see Jacob et al. (2018) for details). Using the Chapman Enskog expansion, it is possible to show that :

$$
f_{\alpha}^{n e q} \approx \underbrace{\frac{Q_{\alpha i j}}{2 c_{s}^{4}} \sum_{\alpha} c_{\alpha i} c_{\alpha j}\left(f_{\alpha}-f_{\alpha}^{e q}\right)}_{f_{\alpha}^{\text {neq } L B M}}=\underbrace{-\frac{\tau \omega_{\alpha}}{2 c_{s}^{2}} Q_{\alpha i j}\left(\frac{\partial \rho u_{j}}{\partial x_{i}}+\frac{\partial \rho u_{i}}{\partial x_{j}}\right)}_{f_{\alpha}^{\text {neq, } D F}}
$$

According to Eq. 7, fneq could be estimated using the local distribution functions $\left(f_{\alpha}^{n e q, L B M}\right)$ or the derivatives of the macroscopic values $\left(f_{\alpha}^{n e q, D F}\right)$, which may be evaluated using second order finite differences. Hence, in order 
to enhance stability while limiting numerical dissipation, $f^{n e q}$ is computed in our model as follows:

$$
f_{\alpha}^{n e q}=\sigma f_{\alpha}^{n e q, L B M}+(1-\sigma) f_{\alpha}^{n e q, D F}
$$

with $\sigma \in[0 ; 1]$.

Treatment of external forces. In order to take source terms $(\tilde{S})$ into account, the right hand side of Eq. 3 can be modified as follows:

$$
f_{\alpha}\left(\mathbf{x}+\mathbf{c}_{\alpha} \Delta t, t+\Delta t\right)-f_{\alpha}(\mathbf{x}, t)=-\frac{\Delta t}{\tau}\left(f_{\alpha}-f_{\alpha}^{e q}\right)+S_{\alpha}(\mathbf{x}, t)
$$

According to Guo et al. (2002), the following development of an external force ( $\mathbf{S}$ non-dimensionalized following $\mathbf{S}=\tilde{\mathbf{S}} \frac{\Delta t^{2}}{\Delta x}$ ) can be more particularly considered in order to accurately recover the Navier-Stokes equations :

$$
\mathbf{S}_{\alpha}(\mathbf{x}, t)=\rho\left(1-\frac{1}{2 \tau}\right) \omega_{\alpha}\left[\frac{\mathbf{c}_{\alpha}-\mathbf{u}}{c_{s}^{2}}+\frac{\mathbf{c}_{\alpha}\left(\mathbf{c}_{\alpha} \cdot \mathbf{u}\right)}{c_{s}^{4}}\right] \cdot \mathbf{S}
$$

The macroscopic velocity is then given by:

$$
\mathbf{u}(\mathbf{x}, t)=\frac{1}{\rho} \sum_{\alpha} \mathbf{c}_{\alpha} f_{\alpha}(\mathbf{x}, t)+\frac{\Delta t}{2 \rho} \mathbf{S}
$$

In the present study, the aerodynamic drag of trees is taken into account by introducing a volumic Forchheimer force $\left(F_{\text {por }}\left[\mathrm{N} \mathrm{m}^{-3}\right]\right)$ designed to account for porous media effects on turbulent flows:

$$
F_{\text {por }}=-\rho \times R \times|\mathbf{u}| \times \mathbf{u} \times \Phi
$$

${ }_{140}$ with: $\left\{\begin{array}{l}R: \text { the drag force coefficient }\left[\mathrm{m}^{-1}\right] \\ \Phi: \text { the ratio of porous media immersed in the volumic cell. }\end{array}\right.$
} 
Hybrid approach. To solve the conservation equations for species (passive scalar) while remaining within the LBM framework, it is possible to use a multidistribution approach. Multidistribution means that an additional superimposed lattice is considered to solve passive scalar transport. Nonetheless, given the correspondence between the LBM and Navier-Stokes approaches, it is also possible to develop a hybrid approach to solve passive scalar transport when basically using the LBM. In such an approach, the mass and momentum conservation equations are solved using the LBM while the species conservation equations are solved using a usual finite volume / finite difference method. This method allows thus to consider only one additional unknown per additional equation. Typically, in ProLB, the species conservation equation is solved using a finite difference vertex centered scheme. A centered scheme using the 18 neighbors defined in the LBM lattice mixed with a first order upwind scheme is used for the advective term whereas a standard centered second order scheme is used for diffusion term.

Boundary conditions. As most of LBM solvers, ProLB uses the immersed boundary method to include solid boundaries. This method decouples the triangular surfacic mesh from the cubic volumic mesh. As each near wall node do not have all its neighbors in the fluid domain, the lattice Boltzmann algorithm cannot be applied. For these particular nodes, macroscopic quantities are computed using an interpolation (Dirichlet condition) or an extrapolation (Neumann condition) between the fluid and the solid boundary, or wall functions. The distribution functions are then reconstructed from equations (4) and (7). This method substantially reduces meshing costs compared to usual unstructured meshes based on surface discretization. 
Large eddy simulation. The LBM is inherently well suited to dynamically solve flows using the LES technique. With LES, the most energy carrying and problem dependent eddies are solved. Conversely, eddies smaller than the spatial filter -typically the grid mesh- are modeled. For this purpose, the Smagorinsky (Smagorinsky, 1963) subgrid viscosity model is commonly used:

$$
\nu_{t}=(C \Delta)^{2}|S|
$$
of Eq. 6 to perform LES.

\section{The CODASC benchmark}

\subsection{Wind tunnel setup and results}

[Figure 1 about here.] Ruck, 2009, 2012) provides detailed reduced-scale measurements of traffic-like induced pollutant concentration next to the walls of a street canyon model. The CODASC deals with different configurations, including different aspect ratios $(\mathrm{H} / \mathrm{W})$, wind incidences and artificial tree plantings. The present study 
focuses on the $H / W=1$ configuration with a wind direction perpendicular to the street canyon axis with and without continuous tree planting.

Reference experiments were carried out in a boundary layer wind tunnel with smooth walls and ceiling. This ceiling was adjusted in order to obtain a zero pressure gradient in the streamwise direction. The cross section is $2 \mathrm{~m}$ large ( $Y$ direction) and $1 \mathrm{~m}$ high ( $Z$ direction).

According to Figure 1(b), small solid elements on the floor were used as roughness to reproduce a typical urban boundary layer mean velocity profile. A 0.3 power law profile for the mean velocity of the boundary layer was achieved. Considering $U_{H}=4.65 \mathrm{~ms}^{-1}$ at the building height $(\mathrm{H})$, the Reynolds number of the test equals $3.7 \times 10^{4}$, so the flow is turbulent. The measured turbulence intensities are characterized by a decreasing -0.36 power law profile with height.

As shown in Figures 1(a) and 1(b), the street canyon model consists of two $\mathrm{H}=0.12 \mathrm{~m}$ high, $\mathrm{L}=10 \mathrm{H}$ long rectangular obstacles made of contiguous blocks of plexiglas. When present, tree crowns were modeled with rectangular volumes made of a fiber like wading material enclosed in suspended metallic lattice cages (Gromke and Ruck, 2009). Trunks were neglected. Different tree crowns types were realized by varying the mass of wading material in the lattice cage. Corresponding porosity properties were experimentally determined and characterized with a normalized pressure loss coefficient $\left(\lambda\left[\mathrm{m}^{-1}\right]\right)$, as follows:

$$
\lambda=\frac{p_{w w}-p_{l w}}{\left(\frac{1}{2} \rho u^{2}\right) d}
$$


${ }_{207}$ with: $\left\{\begin{array}{l}p_{w w}: \text { the windward pressure }[\mathrm{Pa}] \\ p_{l w}: \text { the leeward pressure }[\mathrm{Pa}] \\ \rho: \text { the fluid density }\left[\mathrm{kg} \mathrm{m}^{-3}\right], \\ u: \text { the mean streamwise velocity }\left[\mathrm{m} \mathrm{s}^{-1}\right], \\ d: \text { the streamwise thickness of the wadding material }[\mathrm{m}]\end{array}\right.$

The CODASC database reports measurements for $\lambda=0 \mathrm{~m}^{-1}$ (no tree), ${ }_{210} \lambda=80 \mathrm{~m}^{-1}$ and $\lambda=200 \mathrm{~m}^{-1}$.

The traffic-like pollutant release was modeled using four line sources of equal strength located on the street canyon ground. These sources are more precisely composed of equidistant little openings with high pressure drop to ensure that the release remains unaffected by local pressure fluctuation induced by the street canyon flow. Emissions consisted of a mixture of sulfure hexafluoride (SF6, $Q_{S F 6}=6.5 \mathrm{~cm}^{3} \mathrm{~min}^{-1}$, tracer gas) and dry air $\left(Q_{a i r}=\right.$ $\left.7 \times 10^{3} \mathrm{~cm}^{3} \mathrm{~min}^{-1}\right)$. Mean tracer gas concentrations were measured at $x^{+}=$ $0.04167 \frac{x}{H}$ from street canyon building walls using electron capture detectors. The 700 molar concentration measures $\left(c_{m o l}\right)$ distributed over the 7 horizontal lines available for each wall were normalized as follows:

$$
c^{+}=\frac{C_{m o l} H U_{H}}{Q_{l}}
$$

${ }_{222}$ with: $\left\{\begin{array}{l}c^{+}: \text {the normalized concentration }[-] \\ H \text { : the building height }[\mathrm{m}] \\ U_{H}: \text { the wind velocity at } \mathrm{H}\left[\mathrm{m} \mathrm{s}^{-1}\right] \\ Q_{l}: \text { the emission rate of the line source }\left[\mathrm{m}^{2} \mathrm{~s}^{-1}\right]\end{array}\right.$ 
Experimental measures available in the database were symmeterized. Experimental results show weaker concentration levels on the windward wall (wall B) than on the leeward wall (wall A). This distribution is explained by the formation of a street canyon vortex, which is driven by the above flow (Gromke and Ruck, 2009). The canyon vortex drives pollutant from the street canyon ground towards wall A, and upwards. Part of pollution is then mixed with the above flow at roof level. The other part is re-entrained in a new cycle of the canyon vortex, which explains the presence of pollutant next to wall B. In addition, corner eddies enhance ventilation in the canyon after separation at the lateral edges of block $\mathrm{A}$, which decreases pollution at street canyon ends. In the absence of trees, these typical flow structures of 3D street canyons induce an averaged concentration level on wall $\mathrm{A}$ that is 3.8 times higher than on wall B.

The presence of trees reduces exchanges between the street canyon and the ambient flow. Corner eddies are blocked at the street canyon ends, and the canyon vortex is highlighted weaker in the central part of the street than in the empty street canyon. Velocity is substantially reduced next to wall B. Velocity next to wall A is also reduced. These modifications induce a substantial increase of pollutant concentration levels next to wall $\mathrm{A}$, as well as a decrease of pollutant concentration at wall B. As explained in Gromke et al. (2008), the rotating fluid mass decreases when trees are located in the street, leading to a reduction of the pollutant mass ejected above wall $\mathrm{A}$ to wall B and a decrease of pollutant concentration close to wall B. Thus, higher concentrations are observed in the street canyon with tree planting than in the empty street canyon: the total pollutant increase is about $28 \%$ for 
$\lambda=80 \mathrm{~m}^{-1}$ and $36 \%$ for $\lambda=200 \mathrm{~m}^{-1}$. Additional experiments highlighted no substantial change in wall-averaged pollution concentrations for higher values of $\lambda$.

\subsection{Related studies}

The CODASC benchmark was considered by several studies to assess the performance of different CFD approaches in predicting pollutant dispersion in the presence of trees. Studies often considered the $\mathrm{H} / \mathrm{W}=1$ configuration with a wind incidence perpendicular to the canyon axis (Gromke et al., 2008; Balczó et al., 2009; Salim et al., 2011; Moonen et al., 2013; Gromke and Blocken, 2015a; Vranckx et al., 2015; Kang et al., 2017), or the $\mathrm{H} / \mathrm{W}=0.5$ configuration (Buccolieri et al., 2009, 2011; Abhijith and Gokhale, 2015; Xue and Li, 2017). Table 1 gives an overview of the different CFD studies performed for the $\mathrm{H} / \mathrm{W}=1$ configuration.

[Table 1 about here.]

Studies referenced in Table 1 generally highlighted that simulation is capable of reproducing the main flow and concentration patterns highlighted in the experiment. However, quantitative analysis generally exhibits discrepancies between predictions and measurements. In particular, using steady RANS, the street canyon vortex is generally predicted weaker than observed in the wind tunnel experiments or more detailed approaches (Gromke et al., 2008; Salim et al., 2011; Vranckx et al., 2015). This behavior can be explained by the underprediction of the turbulent kinetic energy at the canyon top, which induces too small shear at the canyon top. Regarding dispersion, studies pointed out the unequal performance of turbulence models (RSM was 
often found to perform better than $k-\varepsilon$ ) and the dependence of predicted concentrations with respect to the choice of the turbulent Schmidt number as well as the limitation of steady state approaches in reproducing the mixing processes, which are intrinsically transient (Gromke et al., 2008; Salim et al., 2011; Gromke and Blocken, 2015b). These reasons could explain the variable behaviors of RANS results in terms of concentrations on walls A and B in the reviewed studies. Nonetheless, overall, studies generally concluded that RANS approaches may constitute an acceptable compromise between prediction accuracy, applicability and computational costs. Using unsteady RANS, Kang et al. (2017) found also a relatively satisfactory agreement between predictions and measurements in terms of flow and concentration patterns for the different tested tree configurations, but concentrations were underestimated for wall A and overestimated for wall B.

When comparing LES to RANS, Salim et al. (2011) found a substantially better performance of LES than that of RANS, especially with respect to the consistency of concentration distributions. The better performance of LES was explained by its ability to reproduce intermittent turbulent fluctuations. LES predictions were found almost satisfactory on wall A, but deviations were still highlighted on wall B (Salim et al., 2011; Moonen et al., 2013). Moreover, Salim et al. (2011) and Moonen et al. (2013) emphasized that LES enables the instantaneous and intermittent behavior of the flow to be analyzed, thus providing information on dispersion processes as well as short term exposure problems.

These advantages of LES involve nonetheless significant additional computational costs with respect to RANS, which may be limiting for its develop- 
ment for urban applications. As a matter of fact, Salim et al. (2011) indicates that performing LES instead of RANS induced an increase of computational costs of one or two orders of magnitude. Hence, given the addressed problem and the conclusions of related studies, this benchmark appears well suited to highlight and discuss the performance of innovative CFD approaches, such as the present LBM LES.

\section{Numerical modeling and performance evaluation}

\subsection{Numerical settings}

To assess the applicability and performance of the LBM LES approach we developed in ProLB for urban pollution issues, this study focuses on a bad case for dispersion: the street canyon perpendicular to the wind incidence. The studied configuration corresponds to the reduced scale $(1: 150) \mathrm{H} / \mathrm{W}=1$ street canyon, containing, or not, continuous avenue-like tree plantings $(\lambda=$ 0,80 and $\left.200 \mathrm{~m}^{-1}\right)$.

\section{[Figure 2 about here.]}

Figure 2(a) depicts the $L_{D} \times W_{D} \times H_{D}=3 \times 2 \times 1 \mathrm{~m}^{3}$ computational domain set for simulation to reproduce the experimental test section. The fetch equals $7 \mathrm{H}$ and the inflow was specified with a velocity inlet condition, as follows (Moonen et al., 2013):

$$
\frac{U(y)}{U_{H}}=\left(\frac{y}{y_{H}}\right)^{k_{u}}
$$


316 with: $\left\{\begin{array}{l}k_{u}=0.3: \text { the power law exponent, } \\ y_{H}=H=0.12 \mathrm{~m}: \text { the canyon height, } \\ U_{H}=4.65 \mathrm{~m} \mathrm{~s}^{-1}: \text { the wind velocity at } \mathrm{y}=\mathrm{H} .\end{array}\right.$

The Synthetic Eddy Method (Pamiès et al., 2009) was used to provide the turbulent contribution of the approaching flow, based on the following profile of turbulence intensity (Moonen et al., 2013):

$$
\frac{I(y)}{I_{H}}=\left(\frac{y+y_{D}}{y_{H}+y_{D}}\right)^{k_{I}}
$$

321 with: $\left\{\begin{array}{l}k_{I}=-0.65: \text { the power law exponent, } \\ I_{H}=14.7 \% \text { : the turbulence intensity at reference height, } \\ y_{d}=-\left(1+\frac{k_{I}}{k_{u}}\right) y_{t k e}, \\ y_{t k e}=0.017 \mathrm{~m}: \text { the vertical position of the center of the shear layer }\end{array}\right.$ A constant pressure condition was set at the outflow. Lateral and top domain boundaries were specified as frictionless walls and the floor was specified as a $3.3 \times 10^{-3} \mathrm{~m}$ high rough floor. The walls of blocks A and B forming the street canyon were assumed smooth (Figure 2(b)). In addition, sponge layers (Xu and Sagaut, 2013) were applied at the top and outlet boundaries (see Figure 2(a)) to absorb waves generated at the initialization of the computation. In these layers, the density is progressively relaxed towards its initial value.

[Figure 3 about here.]

When present, trees were accounted for as simple porous media with 
aerodynamic drag. They were thus modeled by creating porous zones at crown location (Figure 3(a)). The pressure loss coefficient $\left(R\left[\mathrm{~m}^{-1}\right]\right)$ was specified using Eq. 12, according to experimental indications depending on the crown permeability $\left(\lambda\left[\mathrm{m}^{-1}\right]\right)$ following:

$$
R=\frac{\lambda}{2}
$$

[Figure 4 about here.]

Figure 4 displays the mesh used for simulation, which includes five nested refinement zones. Spatial discretization involves $d x=\mathrm{H} / 96$ lattices in the canyon. The corresponding basic time step equals $1.44 \times 10^{-5} \mathrm{~S}$ in order to fulfill CFL and low Mach flow requirements. Overall, $4.1 \times 10^{7}$ grid points are used to mesh the full domain. Simulations were performed on the French GENCI's Occigen supercomputer using 240 cores and run over $25 \mathrm{~s}$ of physical time.

To model pollutant sources, $1.42 \times 1.25 \times 10^{-3} \mathrm{~m}^{2}$ lines sources were created on the domain bottom boundary (Figure 3(b)) and activated at $t=8 \mathrm{~s}$. With a massic emission concentration of $4.786 \times 10^{-3} \mathrm{~kg} \mathrm{~kg}^{-1}$ and a vertical velocity equal to $0.2054 \mathrm{~m} \mathrm{~s}^{-1}$, the mass flow rate of $\mathrm{SF}_{6}$ is $2.094 \times 10^{-6} \mathrm{~kg} \mathrm{~s}^{-1}$. The corresponding mass flow rate of air is $4.375 \times 10^{-4} \mathrm{~kg} \mathrm{~s}^{-1}$. Diffusivity of SF6 was set to $2.3 \times 10^{-5} \mathrm{~m}^{2} \mathrm{~s}^{-1}$ and the subgrid Schmidt number to 0.7. Although an influence of the turbulent Schmidt number was observed on previous RANS studies, only one value of the subgrid Schmidt number was used. Indeed, since a LES model is used the main part of turbulent effects is resolved and only a small part is modeled, which decreases the importance of that numerical parameter. 
According to convergence analysis, the last $10 \mathrm{~s}$ of simulation were kept for post processing and time averaging of results. For physical analysis, velocities $\left(u_{i}\right)$ were normalized $\left(u_{i}^{+}\right)$, as follows:

$$
u_{i}^{+}=\frac{u_{i}}{U_{H}}
$$

and concentration results $\left(c_{m}\right)$ were normalized following:

$$
c^{+}=\frac{c_{m} \times U_{H} \times H}{Q_{S F 6} / l} \times \frac{M_{a i r}}{M_{S F 6}}
$$

360 with: $\left\{\begin{array}{l}c^{+}=: \text {the normalized concentration, } \\ \mathrm{H}=0.12 \mathrm{~m}: \text { the height of the street canyon, } \\ l=1.42 \mathrm{~m}: \text { the length of the line sources, } \\ Q_{S F 6}=1.359 \times 10^{-6} \mathrm{~m}^{3} \mathrm{~s}^{-1}: \text { the volumic flow rate of } \mathrm{SF} 6, \\ M_{a i r}=28.966 \mathrm{~g} \mathrm{~mol}^{-1}: \text { the molar mass of air, } \\ M_{S F 6}=146.055 \mathrm{~g} \mathrm{~mol}^{-1}: \text { the molar mass of SF6. }\end{array}\right.$

\subsection{Grid sensitivity analysis}

In order to analyze the grid influence on the results, the configuration without trees was tested using the grid defined on Sec. 4.1 and a coarser grid with $d x=\mathrm{H} / 48$ in the street canyon. Figure 5 shows the normalized concentration $c^{+}$profiles in front of wall A and B for the two simulated grids and the measurements. A better agreement is observed for the finest grid at wall A, however, there is no significant differences at wall $\mathrm{B}$.

[Figure 5 about here.] 
In order to assess more quantitatively the accuracy of simulation and evaluate the general model performance as recommended in Chang and Hanna (2004); Hanna and Chang (2012); Moonen et al. (2013), several integrated indicators were also estimated:

- the fraction of predictions within a factor of two of observation (FAC2)

- the fractional bias (FB),

- the root normalized mean square error (RNMSE),

- the geometric mean bias (MG),

- the geometric variance (VG),

- and the correlation coefficient (R).

[Table 2 about here.]

Their meaning as well as target values and acceptable range for urban problems according to Chang and Hanna (2004) are synthesized in Table 2. The different indicators computed for the two grids are listed in Table 3. All of them are located in the acceptable range for coarse and fine grid which means that the model is consistent with grid refinement. Furthermore most of the quality metrics computed for the finest grid are closer to the target values than the ones computed for the coarse grid. Since the results presented in Figure 5 and Table 3 are slightly better for the finest grid, this grid with $d x=\mathrm{H} / 96$ in the street canyon was selected for the actual study.

[Table 3 about here.] 


\subsection{Performance evaluation}

Qualitative evaluation.

[Figure 6 about here.]

To evaluate the correspondence between predictions and observations, Figure 6 firstly compares the simulated $w^{+}$field, at $y / \mathrm{H}=0.5$ with $\mathrm{LDV}$ measurements reported in Gromke et al. (2008). Gromke and Ruck (2009) show that wall averaged $c^{+}$is not very sensitive to $\lambda$ value for $\lambda \geq 200 \mathrm{~m}^{-1}$, which means that the flow in the street canyon is quite independent of $\lambda$ in that range of values. From this result, Figure 6(b) compares velocity fields considering $\lambda=200 \mathrm{~m}^{-1}$ for simulation results and $\lambda=250 \mathrm{~m}^{-1}$ for experimental results. Results highlight that predicted and observed velocity distributions are in good agreement, showing both the development of a street canyon vortex, and reduced velocities in and around tree crowns when present, especially next to wall B.

[Figure 7 about here.]

According to Figure 7, which compares $c^{+}$contours on walls $\mathrm{A}$ and $\mathrm{B}$ for the different configurations, reduced velocity tends to increase pollutant concentration in the central part of the street canyon on wall A and decreases it on wall $\mathrm{B}$, although it is less obvious on simulation results. More specifically, maximum concentration occurs for the densest tree configuration on wall $\mathrm{A}\left(c_{\max , e x p}^{+} \approx 60\right.$ and $c_{\max , \mathrm{sim}}^{+} \approx 70$ for $\lambda=200 \mathrm{~m}^{-1}$ vs. $c_{\max , \exp }^{+} \approx 40$ and $c_{\text {max,sim }}^{+} \approx 45$ for $\lambda=0 \mathrm{~m}^{-1}$ ), and concentration decreases or remains equivalent on wall $\mathrm{B}$ when dense tree crowns are present $\left(c_{\text {max,exp }}^{+} \approx 5\right.$ and 
413

$c_{\text {max }, \text { sim }}^{+} \approx 25$ for $\lambda=200 \mathrm{~m}^{-1}$ vs. $c_{\text {max }, \text { exp }}^{+} \approx 10$ and $c_{\text {max }, \text { sim }}^{+} \approx 20$ for $\left.\lambda=0 \mathrm{~m}^{-1}\right)$.

Figure 7 also highlights the effects of the finite length of the street canyon. The formation of the corner vortices induces a decrease of concentration distribution on walls $\mathrm{A}$ and $\mathrm{B}$ from $y / \mathrm{H}=0$ to the street canyon ends. According to simulation, $c^{+}$values are on average more than 2.3 times higher in the fifth central part of wall $\mathrm{A}(|y / H|<1)$ than on the rest of the wall when trees are present. This ratio equals 2.1 without trees. Because of the blocking effect of trees, concentration increases on wall A and decreases on wall B for $|y / \mathrm{H}|>2.5$. Such a trend is also highlighted in experimental results, although the measured $c^{+}$show smoother gradients in the $y$ direction in the central part of the canyon than simulated, especially when trees are present. Simulation results also exceed experimental data on wall B at this location.

On average, comparing simulation results to measurements, results show that simulation satisfactorily predicts wall averaged concentration on wall A $\overline{\left(c_{\text {sim }, A}^{+}\right.}=18.6$ vs $\left.\overline{c_{e x p, A}^{+}}=19.6\right)$, but overpredicts that of wall $\mathrm{B}\left(\overline{c_{\text {sim }, B}^{+}}=9.6\right.$ vs $\left.\overline{c_{\text {exp }, B}^{+}}=5.4\right)$ in the absence of trees. Including trees in the modeling also induce a limited relative modification of concentration levels on walls $\mathrm{A}$ and B compared to the experiment: $\overline{c_{\text {sim, } A}^{+}}$increases by $18 \%$ and $23 \%$, and $\overline{c_{s i m, B}^{+}}$ decreases by $11 \%$ and $15 \%$, for $\lambda=80$ and $200 \mathrm{~m}^{-1}$ respectively according to current results, whereas $\overline{c_{\exp , A}^{+}}$increases by $41 \%$ and $58 \%$, and $\overline{c_{e x p, B}^{+}}$ decreases by $37 \%$ and $49 \%$, for $\lambda=80$ and $200 \mathrm{~m}^{-1}$ respectively according to Gromke et al. (2008) and Gromke and Ruck (2009).

[Figure 8 about here.] 
To compare observations and predictions in more details, Figure 8 provides $c^{+}$profiles at different locations on walls $\mathrm{A}$ and $\mathrm{B}$. As expected from Figure 8, simulations and observations match quite well on wall A with the exception of $y / \mathrm{H}=1.26$, where simulation results are about the half of the measured values for the densest tree configuration. Conversely to wall $\mathrm{A}$, simulation results exceed measurements on wall B, especially next to the floor, with a relative deviation often greater than a factor of 2 . The same behavior was observed in the LES results of Salim et al. (2011) for wall B in the presence of tree plantings. Current simulation results even show an inverse effect of trees at $y / \mathrm{H}=0.05$ and 0.45 compared to measurements. With respect to Moonen et al. (2013), current results are in closer agreement with experimental data in the central part of the street canyon, but deviate more around $y / \mathrm{H}=1.26$.

\section{Quantitative evaluation.}

[Figure 9 about here.]

Figure 9 summarizes the quality metrics defined in Table 2 computed for both or only wall A and wall $\mathrm{B}$, along with values available in literature that were obtained by Moonen et al. (2013) and Kang et al. (2017). Results show that current quality metrics mostly belong to the recommendation ranges when considering both walls A and B. However, wall-by-wall results analysis confirms previous statements: quality metrics are mostly close to target values for wall A but half of indicators fall out of recommendation ranges for wall B, for which reference concentrations used for scaling are low. Also, the denser the crowns, the further apart the predictions from experimental 
data are. The same observations holds for previously existing simulations (Moonen et al., 2013; Kang et al., 2017).

More specifically, VG and R generally belong to the recommendation ranges. These results show that predictions and experimental data are well correlated, which is a necessary condition to guarantee the effective performance of the model. FAC2 and RNMSE also mostly belong to the recommendation ranges. Deviation occurs mainly on wall B for the densest tree configuration. The good performance of FAC2 is an important information regarding the general performance of the model as this value is not very influenced by outliers. RNMSE confirms previous conclusions relative to the acceptability of the relative scatter. FB and MG show the least satisfactory agreement between numerical and experimental data, as prediction fall out of recommendation ranges when trees are present and for wall B. These results characterize a systematic error. The negative values of FB on wall $\mathrm{B}$ clearly reflect the overprediction of concentration at this location. Nonetheless, according to Figure 9, present simulations show an overall comparable performance as most accurate literature references.

Summary. Hence, similarly to most of reference computational studies, simulation results satisfactorily agree with experimental data in terms of trends and concentration levels without trees and on wall A, which is the most critical location in terms of exposure problems as the highest concentration levels occur there. Predictions and observations deviate more on wall B, which shows lower concentration levels. On average, effects of trees appear accurately reproduced as simulation shows increased concentration levels on wall A and slightly reduced concentration levels on wall B when trees are 
present, but trees are found more influential in the experiments.

Differences between predictions and observations may be explained by some modeling assumptions, which do not strictly correspond to the experimental configuration. In particular, deviation might be explained, at least partly, by the fact that a smooth boundary condition was specified at walls $\mathrm{A}$ and $\mathrm{B}$ in the numerical model, which appears not to be exactly the case in the experiment according to Figure 1(b): joints between blocks as well as taps modify the Plexiglas wall surface. Also, roughness on the street canyon floor differs from around in the experiment while a uniformly rough floor was assumed in the numerical model. In addition, regarding the effect of trees, the metallic lattice is not taken into account in the present numerical model whereas wind tunnel experiments found that the presence of the empty lattice cage induces an increase of pollutant concentration by $18 \%$ on wall $\mathrm{A}$ and a decrease of $16 \%$ on wall $\mathrm{B}$ with respect to the fully empty case. Thus, the accumulation of these apparently small differences in the geometric models could lead to significant deviation in concentration results by modifying flow properties in the street canyon.

\section{Physical analysis of flow and concentration fields}

Results presented in Section 4.3 pointed out effects of trees as well as of the finite length of the canyon on the distribution and level of pollutant concentration at walls. These modifications being due to changes in air flows in the street canyon, this section analyses the flow field induced by the different configurations to highlight basic turbulent dispersion mechanisms as made possible by LES. 


\subsection{Air flow structures}

[Figure 10 about here.]

According to the mean velocity streamlines shown in Figure 10, the flow resistance induced by tree crowns limits the formation of the street canyon vortex, blocks corner eddies and modifies the flow structure in the lower part of the canyon. In the presence of trees, the street canyon vortex is deformed and its center shifted towards wall B. This alteration of the vortex structure around $y / \mathrm{H}=0$ leaves more room to a weak secondary recirculation in the bottom upstream part of the canyon, and modifies the mixing layer at its top.

[Figure 11 about here.]

The effect of trees on turbulent structures can be more clearly identified in Figure 11, which compares instantaneous 3D isocontours of $\mathrm{Q}$ criterion $\left(Q=5 \times 10^{4}\right)$ to identify coherent structures within the street canyon and in the mixing layers bounding it. Results show that, after separation at the top and side leading edges of block $\mathrm{A}$, the turbulent structures develop differently downstream in the street canyon depending on the configuration. Coloration by $w^{+}$suggests that a $3 \mathrm{D}$ canyon and corner vortices develop, with eddies filling all the street canyon volume in the absence of trees. In the presence of trees, these turbulent structures are no more visible next to wall A due to the blocking effect of trees.

[Figure 12 about here.] 
To evaluate more quantitatively the effects of trees on turbulence, Figure 12 depicts boxplots of instantaneous velocity components normalized by $U_{H}\left(u^{+}, v^{+}, w^{+}\right)$in the center of the street canyon $(y / \mathrm{H}=0)$ or at the street canyon ends $(y / \mathrm{H}=5)$. Two points are more particularly emphasized in Figure 12(a): point M is located in the middle of the street canyon and point A is located in the bottom leeward part of the street canyon, i.e. where concentrations are the highest. The central line of boxplots indicate the median value, the circle corresponds to the mean value, boxplot edges are the 25 and 75 percentiles and the ends of the whiskers represent the extreme values. Crosses are related to outliers.

As expected, results show that mean velocity and fluctuations are generally higher at $y / \mathrm{H}=5$ than at $y / \mathrm{H}=0$ for both points $\mathrm{M}$ and $\mathrm{A}$. The direct lateral interactions with the general boundary layer, induces standard deviations that are generally more than 3 times higher at $y / \mathrm{H}=5$ than at $y / \mathrm{H}=0$. Without trees, the corner vortex also induces relatively high mean velocities at point $\mathrm{M}\left(v=-1.25 \mathrm{~m} \mathrm{~s}^{-1}\right)$ as the flow enters the street canyon from its sides. On the contrary, velocity at point $\mathrm{M}$ is rather low at $y / \mathrm{H}=0$, because the canyon vortex is almost centered in the canyon. Being located on the edge of this vortex, point A shows higher velocities $\left(u=-0.35 \mathrm{~m} \mathrm{~s}^{-1}\right)$.

Regarding the effects of trees, Figure 12 confirms previous results: trees significantly alter mean velocities as well as fluctuations at point $\mathrm{M}$, which is located in tree crowns. More specifically, at $y / \mathrm{H}=0$, the alteration of the canyon vortex due to the presence of dense trees reduces $u$ by a factor of 7 at point $\mathrm{M}$, i.e. down to nearly zero (with a change of sign), while $w$ is increased by a factor of 17, making $w$ non null. Corresponding standard deviations are 
divided by a factor about 5 for both components. Regarding point A, $u$ is divided by a factor of 6 and $w$ is reduced by a factor of 2 . Standard deviations are less altered by trees than for point M, as they are reduced by less than $15 \%$ and $30 \%$ respectively for $u$ and $w$. Standard deviation is even increased by $35 \%$ for $v$. At $y / \mathrm{H}=5$, tree crowns limit $v$ at point $\mathrm{M}$ by a factor of 3 and $u$ by a factor of 12 (with a change of sign). Corresponding standard deviations are reduced by a factor of 1.6. Considering point $\mathrm{A}, u$ and $w$ are reduced by less than a factor of 2 , and $v$ by a factor of 6 (with a change of sign). Standard deviations are only reduced by $10 \%$ for $u$ and $w$ and by $20 \%$ for $v$.

Hence, these results highlight that trees alter velocities at points $\mathrm{A}$ and $\mathrm{M}$, which is related to the alteration of the general flow structures in the middle and at the end of the canyon, i.e. in the canyon and the corner vortices. However, while both mean velocities and fluctuations at point $\mathrm{M}$ are generally reduced because of tree crowns, effects of trees on fluctuations at point $\mathrm{A}$ are less straightforward.

\subsection{Effect of turbulence on dispersion}

[Figure 13 about here.]

According to Figures 10 and 7, clean air entering the canyon from above in front of the windward wall and from the sides of the street canyon pushes pollutant towards the central leeward part of the canyon. A jet raises there and drives the pollutant out, especially when trees are present. Focusing on this critical part of the street canyon, Figure 13 clearly shows how the reduction of turbulence and velocities induced by tree crowns affect pollutant 
dispersion. The reduced ventilation potential of the street canyon increases pollutant concentration levels within it. More precisely, while pollutant is driven by the main street canyon vortex from line sources to the top mixing layer in the absence of trees, porous zones favor the diffusion and the residence of pollutant inside the street canyon. The less porous the crown, the higher the concentration. Pollutant is especially retained below and inside tree crowns, as well as in the leeward part of the street canyon. The flow developing there extends further above wall $\mathrm{A}$ at the canyon top and in the mixing layer, which contaminates the separation bubble above block A.

\section{[Figure 14 about here.]}

Similarly to Section 5.1, Figure 14 displays boxplots of instantaneous $c^{+}$at $y / \mathrm{H}=0$ or at the street canyon ends $(y / \mathrm{H}=5)$ for a point $\mathrm{M}$ and $\mathrm{A}$ in order to analyze more quantitatively the effects of trees on turbulent dispersion processes. As opposed to velocity, and as highlighted in Section 4.3, results show that concentration levels are about one order of magnitude higher at $y / \mathrm{H}=0$ than at $y / \mathrm{H}=5$ for point $\mathrm{M}$, where concentration at $y / \mathrm{H}=$ 5 are very low. This difference is also substantial at point A, for which concentration levels at $y / H=5$ are also low. The denser the crowns, the greater the difference. Standard deviations are also significantly higher at $y / \mathrm{H}=0$ than at $y / \mathrm{H}=5$.

Still conversely to velocity, the influence of trees on concentration differs depending on the considered location: tree crowns increase concentration levels at $y / \mathrm{H}=0$ and slightly decrease it at $y / \mathrm{H}=5$ for points $\mathrm{A}$ and $\mathrm{M}$. More specifically, at $y / \mathrm{H}=0$, dense tree crowns increase mean concentration 
by a factor of 2 at point $\mathrm{M}$ and 1.8 at point $\mathrm{A}$ compared with the configuration without trees. Corresponding standard deviations are increased by a factor of 2.7 and 1.3 respectively. Reached peak values are very high as $c^{+}$ substantially exceeds 100 several times when trees are present, even if mean concentrations may be less than the half of this peak value.

Hence, the reduction of velocities by trees tends to increase mean concentration and associated fluctuations at $y / \mathrm{H}=0$, i.e. where concentration levels are the highest. Very high instantaneous concentrations might occur at point $\mathrm{A}$, where the mean concentration levels significantly exceed the median values. This means that instantaneous concentration can be much higher than the mean value, which can be prejudicial for people's health in case of short time exposure to some specific pollutants.

\section{Concluding remarks}

This study assesses the performance of a LBM-LES approach in predicting pollutant dispersion in street canyons in the presence or absence of trees. Simulation results compare very satisfactorily to state-of-the-art results obtained for the same benchmark configurations using Navier-Stokesbased LES approaches. Predictions exhibit a very satisfactory agreement with experimental data on wall $\mathrm{A}$, which is critical as this wall shows the highest concentration levels, while larger differences are observed on wall $\mathrm{B}$ (as in all previously reported numerical results), where concentration levels are relatively low. The general effect of crowns on dispersion observed in the experiment is well reproduced by simulations, but with less accuracy when decreasing crown permeability. This deviation may be explained, at 
least partly, by differences between the numerical and experimental models, as the flow, and thus dispersion processes, are very sensitive to geometric details at this scale.

Further, present results show that the developed unsteady high-fidelity approach is valuable to predict and understand air flows and dispersion processes and thus the local urban breathability (Panagiotou et al., 2013). In particular, effects of tree crowns on the development of usual canyon and corner vortices have been studied in detail. The analysis of the results especially pointed out the alteration of the general mean flow structures as well as of intermittent processes at different locations in the street canyon. Such results enable turbulent dispersion to be better predicted, and rapid phenomena that are critical for short term exposure issues to be identified.

Hence, this study shows that the LBM-LES yields state-of-the-art results, while allowing the use of very fine spatial and temporal resolutions thanks to its computational efficiency. In addition, the use of embedded uniform meshes with immersed boundary conditions allows to handle complex geometries in a very easy way, which reduces pre-processing efforts for urban problems. Therefore, based on present results, this approach appears well suited to further study dispersion in realistic urban environments including complex building and street geometries, different atmospheric stability states or even moving bodies such as motorized engines or people.

\section{Acknowledgments}

The authors acknowledge support from several French institutions. This work was supported by the French project CLIMB, with the financial sup- 
port of BPI France (Project No. P3543-24000), in the framework of the program "Investissement d'Avenir: Calcul Intensif et Simulation Numérique". This work was performed using HPC resources from GENCI-TGCC/CINES (Grant 2018-A0032A07679).

The authors also sincerely thank the Laboratory of Building and Environmental Aerodynamics of the Karlsruhe Institute of Technology for providing the CODASC data.

\section{References}

Abhijith, K., Gokhale, S., Sep. 2015. Passive control potentials of trees and on-street parked cars in reduction of air pollution exposure in urban street canyons. Environmental Pollution 204, 99-108.

Abhijith, K., Kumar, P., Gallagher, J., McNabola, A., Baldauf, R., Pilla, F., Broderick, B., Di Sabatino, S., Pulvirenti, B., Aug. 2017. Air pollution abatement performances of green infrastructure in open road and builtup street canyon environments - A review. Atmospheric Environment 162, $71-86$.

Ahmad, K., Khare, M., Chaudhry, K., Sep. 2005. Wind tunnel simulation studies on dispersion at urban street canyons and intersections - A review. Journal of Wind Engineering and Industrial Aerodynamics 93 (9), 697-717.

Ahmad, N. H., Inagaki, A., Kanda, M., Onodera, N., Aoki, T., Jun. 2017. Large-Eddy Simulation of the gust index in an urban area using the Lattice Boltzmann Method. Boundary-Layer Meteorology 163 (3), 447-467. 
Balczó, M., Gromke, C., Ruck, B., May 2009. Numerical modeling of flow and pollutant dispersion in street canyons with tree planting. Meteorologische Zeitschrift 18 (2), 197-206.

Blocken, B., Jun. 2014. 50 years of Computational Wind Engineering: Past, present and future. Journal of Wind Engineering and Industrial Aerodynamics 129, 69-102.

Blocken, B., Sep. 2015. Computational Fluid Dynamics for urban physics: Importance, scales, possibilities, limitations and ten tips and tricks towards accurate and reliable simulations. Building and Environment 91, 219-245.

Britter, R. E., Hanna, S. R., 2003. Flow and dispersion in urban areas. Annual Review of Fluid Mechanics 35 (1), 469-496.

Buccolieri, R., Gromke, C., Di Sabatino, S., Ruck, B., Sep. 2009. Aerodynamic effects of trees on pollutant concentration in street canyons. Science of The Total Environment 407 (19), 5247-5256.

Buccolieri, R., Salim, S. M., Leo, L. S., Di Sabatino, S., Chan, A., Ielpo, P., de Gennaro, G., Gromke, C., Mar. 2011. Analysis of local scale tree-atmosphere interaction on pollutant concentration in idealized street canyons and application to a real urban junction. Atmospheric Environment 45 (9), 1702-1713.

Chang, J. C., Hanna, S. R., Sep. 2004. Air quality model performance evaluation. Meteorology and Atmospheric Physics 87 (1-3).

Chen, S., Doolen, G. D., 1998. Lattice Boltzmann method for fluid flows. Annual Review of Fluid Mechanics 30 (1), 329-364. 
CS, 2016. Main scientific document of LaBS (ProLB). Tech. rep.

CS, 2018. ProLB.

\section{URL http://www.prolb-cfd.com/}

Fan, Z., Qiu, F., Kaufman, A., Yoakum-Stover, S., 2004. GPU cluster for high performance computing. In: Supercomputing, 2004. Proceedings of the ACM/IEEE SC2004 Conference. IEEE, pp. 47-47.

Gromke, C., Blocken, B., Jan. 2015a. Influence of avenue-trees on air quality at the urban neighborhood scale. Part I: Quality assurance studies and turbulent Schmidt number analysis for RANS CFD simulations. Environmental Pollution 196, 214-223.

Gromke, C., Blocken, B., Jan. 2015b. Influence of avenue-trees on air quality at the urban neighborhood scale. Part II: Traffic pollutant concentrations at pedestrian level. Environmental Pollution 196, 176-184.

Gromke, C., Buccolieri, R., Di Sabatino, S., Ruck, B., Dec. 2008. Dispersion study in a street canyon with tree planting by means of wind tunnel and numerical investigations - Evaluation of CFD data with experimental data. Atmospheric Environment 42 (37), 8640-8650.

Gromke, C., Ruck, B., 2007. Influence of trees on the dispersion of pollutants in an urban street canyon - Experimental investigation of the flow and concentration field. Atmospheric Environment 41 (16), 3287 - 3302.

Gromke, C., Ruck, B., Apr. 2009. On the impact of trees on dispersion processes of traffic emissions in street canyons. Boundary-Layer Meteorology 131 (1), 19-34. 
Gromke, C., Ruck, B., Jul. 2012. Pollutant concentrations in street canyons of different aspect ratio with avenues of trees for various wind directions. Boundary-Layer Meteorology 144 (1), 41-64.

Guo, Z., Shu, C., 2013. The Lattice Boltzmann Method and its applications in engineering. World Scientific.

Guo, Z., Zheng, C., Shi, B., Apr. 2002. Discrete lattice effects on the forcing term in the lattice Boltzmann method. Physical Review E 65 (4).

Hanna, S., Chang, J., May 2012. Acceptance criteria for urban dispersion model evaluation. Meteorology and Atmospheric Physics 116 (3-4), 133146.

Jacob, J., Malaspinas, O., Sagaut, P., 2018. A new Hybrid Recursive Regularized Bathnagar-Gross-Krook collision model for Lattice-BoltzmannMethod based Large-Eddy simulation. submitted.

Jacob, J., Sagaut, P., 2018. Wind comfort assessment by means of large eddy simulation with lattice Boltzmann method in full scale city area. Building and Environment 139, 110 - 124.

Janhäll, S., Mar. 2015. Review on urban vegetation and particle air pollution - Deposition and dispersion. Atmospheric Environment 105, 130-137.

Jeanjean, A. P., Buccolieri, R., Eddy, J., Monks, P. S., Leigh, R. J., Mar. 2017. Air quality affected by trees in real street canyons: The case of Marylebone neighbourhood in central London. Urban Forestry \& Urban Greening 22, 41-53. 
Kang, G., Kim, J.-J., Kim, D.-J., Choi, W., Park, S.-J., Nov. 2017. Development of a computational fluid dynamics model with tree drag parameterizations: Application to pedestrian wind comfort in an urban area. Building and Environment 124, 209-218.

King, M.-F., Khan, A., Delbosc, N., Gough, H. L., Halios, C., Barlow, J. F., Noakes, C. J., Nov. 2017. Modelling urban airflow and natural ventilation using a GPU-based lattice-Boltzmann method. Building and Environment $125,273-284$.

KIT, Aug. 2017. CODASC: COncentration DAta of Street Canyons - Karlsruhe Institute of Technology, Laboratory of Building \& Environmental Aerodynamics.

URL http://www.windforschung.de/CODASC.htm

Krüger, T., Kusumaatmaja, H., Kuzmin, A., Shardt, O., Silva, G., Viggen, E., 2017. The Lattice Boltzmann Method. Principles and Practice. Springer.

Lateb, M., Meroney, R., Yataghene, M., Fellouah, H., Saleh, F., Boufadel, M., Jan. 2016. On the use of numerical modelling for near-field pollutant dispersion in urban environments - A review. Environmental Pollution 208, $271-283$.

M2P2, 2018. LaBS.

URL

http: //www.m2p2.fr/valorisation-6/transferttechnologique-labs-3202.htm 
Margheri, L., Sagaut, P., Nov. 2016. A hybrid anchored-ANOVA POD/Kriging method for uncertainty quantification in unsteady highfidelity CFD simulations. Journal of Computational Physics 324, 137-173.

Mons, V., Margheri, L., Chassaing, J.-C., Sagaut, P., Oct. 2017. Data assimilation-based reconstruction of urban pollutant release characteristics. Journal of Wind Engineering and Industrial Aerodynamics 169, 232250.

Moonen, P., Defraeye, T., Dorer, V., Blocken, B., Carmeliet, J., Sep. 2012. Urban Physics: Effect of the micro-climate on comfort, health and energy demand. Frontiers of Architectural Research 1 (3), 197-228.

Moonen, P., Gromke, C., Dorer, V., Aug. 2013. Performance assessment of Large Eddy Simulation (LES) for modeling dispersion in an urban street canyon with tree planting. Atmospheric Environment 75, 66-76.

Obrecht, C., Kuznik, F., Merlier, L., Roux, J.-J., Tourancheau, B., Aug. 2015. Towards aeraulic simulations at urban scale using the lattice Boltzmann method. Environmental Fluid Mechanics 15 (4), 753-770.

Pamiès, M., Weiss, P.-E., Garnier, E., Deck, S., Sagaut, P., Apr. 2009. Generation of synthetic turbulent inflow data for large eddy simulation of spatially evolving wall-bounded flows. Physics of Fluids 21 (4), 045103.

Panagiotou, I., Neophytou, M. K.-A., Hamlyn, D., Britter, R. E., 2013. City breathability as quantified by the exchange velocity and its spatial variation in real inhomogeneous urban geometries: An example from central London urban area. Science of The Total Environment 442, 466 - 477. 
Qiu, F., Zhao, Y., Fan, Z., Wei, X., Lorenz, H., Wang, J., Yoakum-Stover, S., Kaufman, A., Mueller, K., 2004. Dispersion simulation and visualization for urban security. In: Visualization, 2004. IEEE. IEEE, pp. 553-560.

Salim, S. M., Cheah, S. C., Chan, A., Sep. 2011. Numerical simulation of dispersion in urban street canyons with avenue-like tree plantings: Comparison between RANS and LES. Building and Environment 46 (9), 17351746.

Salim, S. M., Ong, K. C., 2013. Performance of RANS, URANS and LES in the Prediction of Airflow and Pollutant Dispersion. In: Kim, H. K., Ao, S.I., Rieger, B. B. (Eds.), IAENG Transactions on Engineering Technologies. Vol. 170. Springer Netherlands, Dordrecht, pp. 263-274, dOI: 10.1007/97894-007-4786-9_21.

Santiago, J.-L., Martilli, A., Martin, F., Mar. 2017. On Dry Deposition Modelling of Atmospheric Pollutants on Vegetation at the Microscale: Application to the Impact of Street Vegetation on Air Quality. Boundary-Layer Meteorology 162 (3), 451-474.

Shan, X., Yuan, X.-F., Chen, H., 2006. Kinetic theory representation of hydrodynamics: a way beyond the Navier-Stokes equation. J. Fluid Mech. $550,413-441$.

Smagorinsky, J., 1963. General circulation experiments with the primitive equations. 1. The basic experiment. Monthly Weather Review 91, 99-164.

Succi, S., 2001. The lattice Boltzmann equation for fluid dynamics and beyond. Oxford University Press, Oxford. 
Tabor, G., Baba-Ahmadi, M., Apr. 2010. Inlet conditions for large eddy simulation: A review. Computers \& Fluids 39 (4), 553-567.

Tominaga, Y., Stathopoulos, T., Apr. 2011. CFD modeling of pollution dispersion in a street canyon: Comparison between LES and RANS. Journal of Wind Engineering and Industrial Aerodynamics 99 (4), 340-348.

Tominaga, Y., Stathopoulos, T., Nov. 2013. CFD simulation of near-field pollutant dispersion in the urban environment: A review of current modeling techniques. Atmospheric Environment 79, 716-730.

Tominaga, Y., Stathopoulos, T., Aug. 2016. Ten questions concerning modeling of near-field pollutant dispersion in the built environment. Building and Environment 105, 390-402.

Vardoulakis, S., Fisher, B. E., Pericleous, K., Gonzalez-Flesca, N., 2003. Modelling air quality in street canyons: A review. Atmospheric environment 37 (2), 155-182.

Vranckx, S., Vos, P., Maiheu, B., Janssen, S., Nov. 2015. Impact of trees on pollutant dispersion in street canyons: A numerical study of the annual average effects in Antwerp, Belgium. Science of The Total Environment $532,474-483$.

World Heath Organization, 2016. WHO Global Urban Ambient Air Pollution Database (update 2016).

URL http://www.who.int/sustainable-development/transport/ health-risks/air-pollution/en/ 
834 World Heath Organization, 2018. Air pollution.

835

836

837

URL http://www.who.int/sustainable-development/transport/ health-risks/air-pollution/en/

Xu, H., Sagaut, P., 2013. Analysis of the absorbing layers for the weakly-compressible lattice Boltzmann methods. Journal of Computational Physics 245, $14-42$.

Xue, F., Li, X., Apr. 2017. The impact of roadside trees on traffic released PM 10 in urban street canyon: Aerodynamic and deposition effects. Sustainable Cities and Society 30, 195-204. 


\section{List of Figures}

1 CODASC experimental model CCODASC, KIT. . . . . . . 42

2 Numerical model: general dimensions and boundary conditions 43

3 Specific dimensions of the virtual street canyon . . . . . . . . 44

4 Computational mesh (5 refinement levels, $d x=\mathrm{H} / 96)$. . . . 45

5 Comparisons between measured $(\boldsymbol{\square})$ and simulated $c^{+}$vertical profiles for the coarse (—) and fine (—) grid .... . 46

6 Comparison between measured (left - (Gromke et al., 2008)) and simulated (right) $w^{+}$contours in the plane $y / \mathrm{H}=0.5 . .47$

$7 c^{+}$contours for $\lambda=0 \mathrm{~m}^{-1}, \lambda=80 \mathrm{~m}^{-1}$ and $\lambda=200 \mathrm{~m}^{-1}$, experimental Figure (C $(\mathrm{KIT}, 2017)$. . . . . . . . . . . . . . 48

8 Comparison between measured and simulated $c^{+}$vertical profiles 49

9 Comparison of quality metrics obtained using ProLB with results of Moonen et al. (2013) and Kang et al. (2017) . . . . . . 50

10 Simulated mean velocity streamlines: 3D view (left) and in a 2D plane (right) . . . . . . . . . . . . . . 51

$113 \mathrm{D}$ isocontours of the $\mathrm{Q}$ criterion $\left(Q=5 \times 10^{4}\right)$ colored by $w^{+}$, the facing section correspond to $y / H=-5 \ldots$. . . . . 52

12 Boxplot of instantaneous velocity components at points $\mathrm{A}$ and M ...................... 53

13 Simulated instantaneous (left) and mean (right) $c^{+}$fields in the $y / \mathrm{H}=0$ plane. . . . . . . . . . . . . . . . . 54

14 Boxplot of instantaneous concentration $\left(c^{+}\right) \ldots \ldots . . . . \quad 55$ 


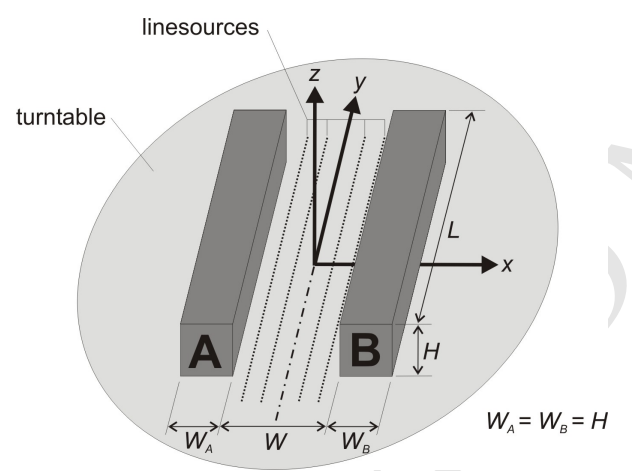

(a) Identification of blocks A and B and coordinate system
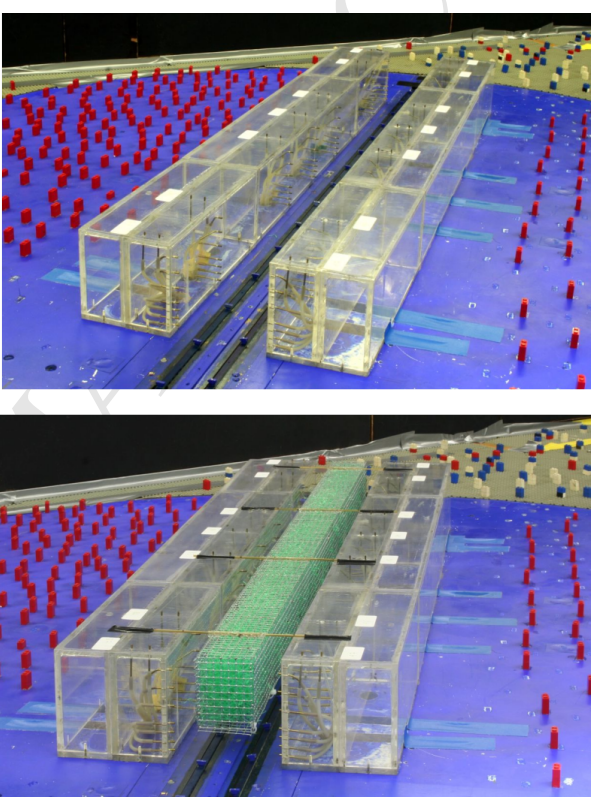

(b) Pictures of the experimental setup

Figure 1: CODASC experimental model (C)CDASC, KIT. 


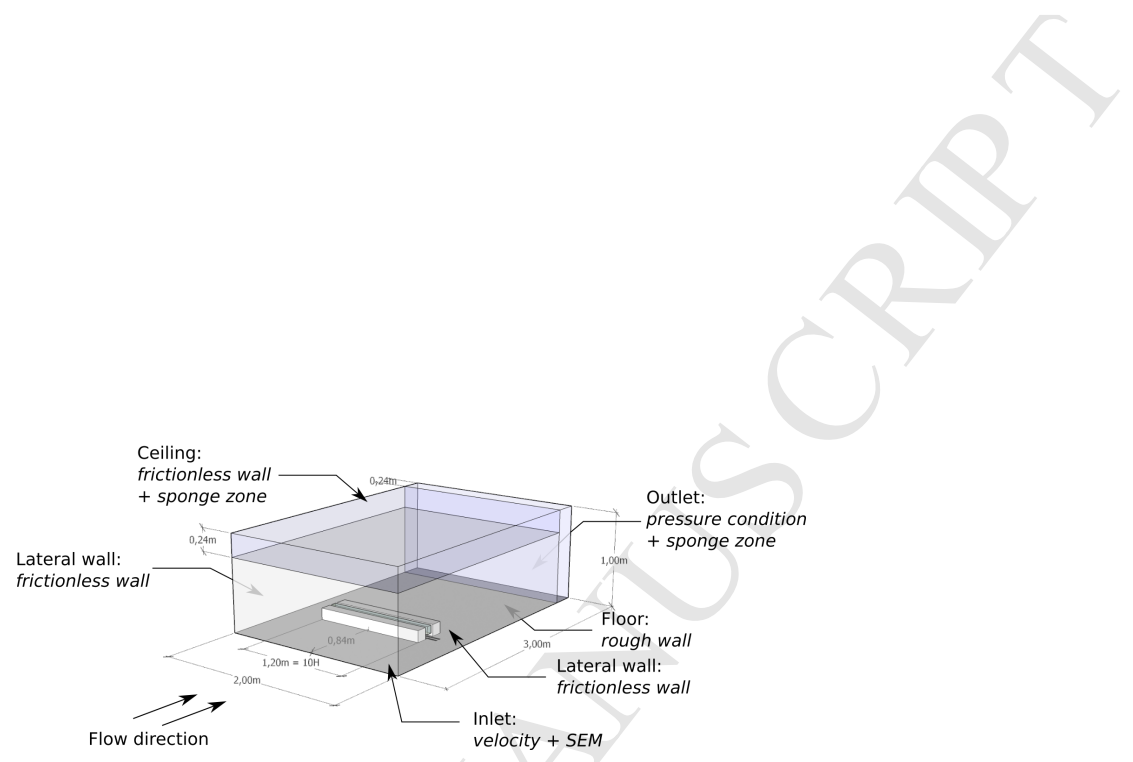

(a) General model settings

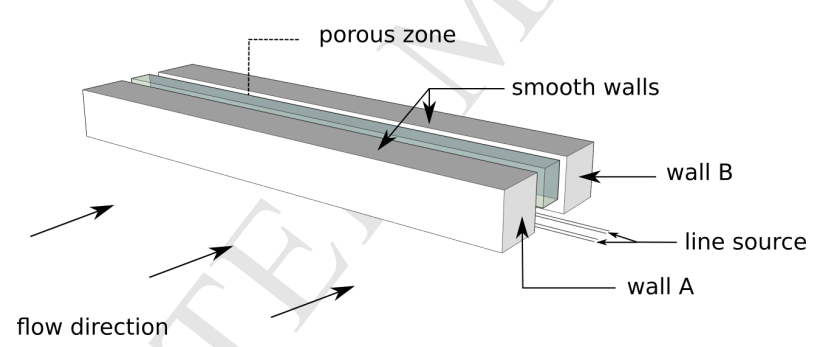

(b) Street canyon model

Figure 2: Numerical model: general dimensions and boundary conditions 


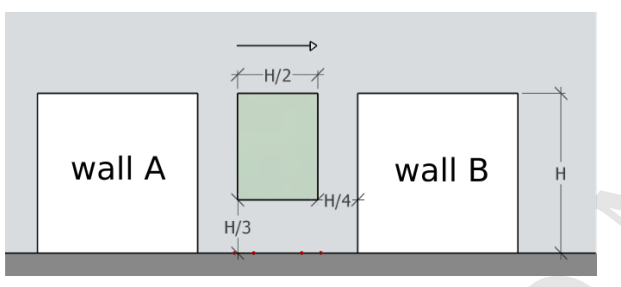

(a) Location of trees

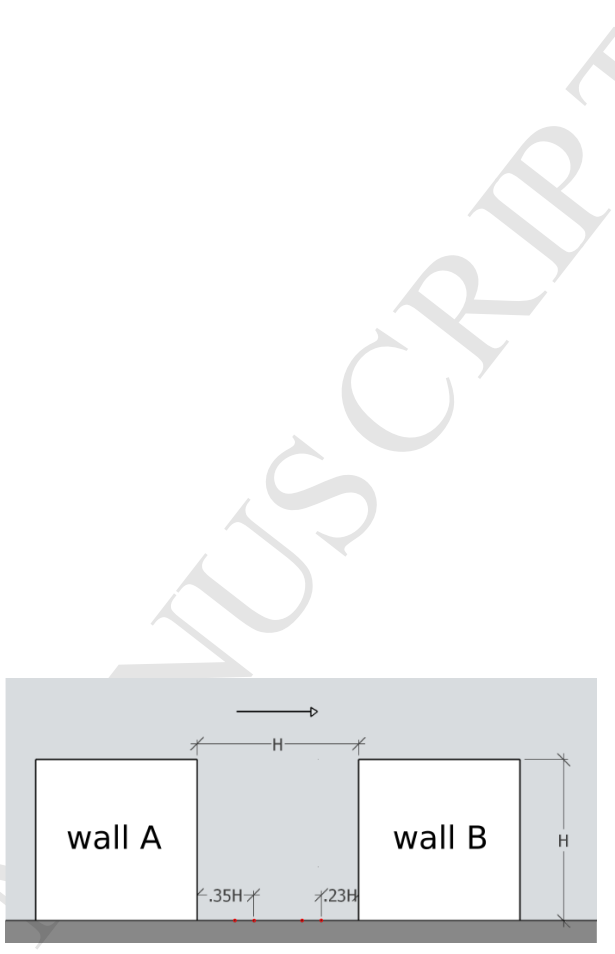

(b) Location of source lines

Figure 3: Specific dimensions of the virtual street canyon 


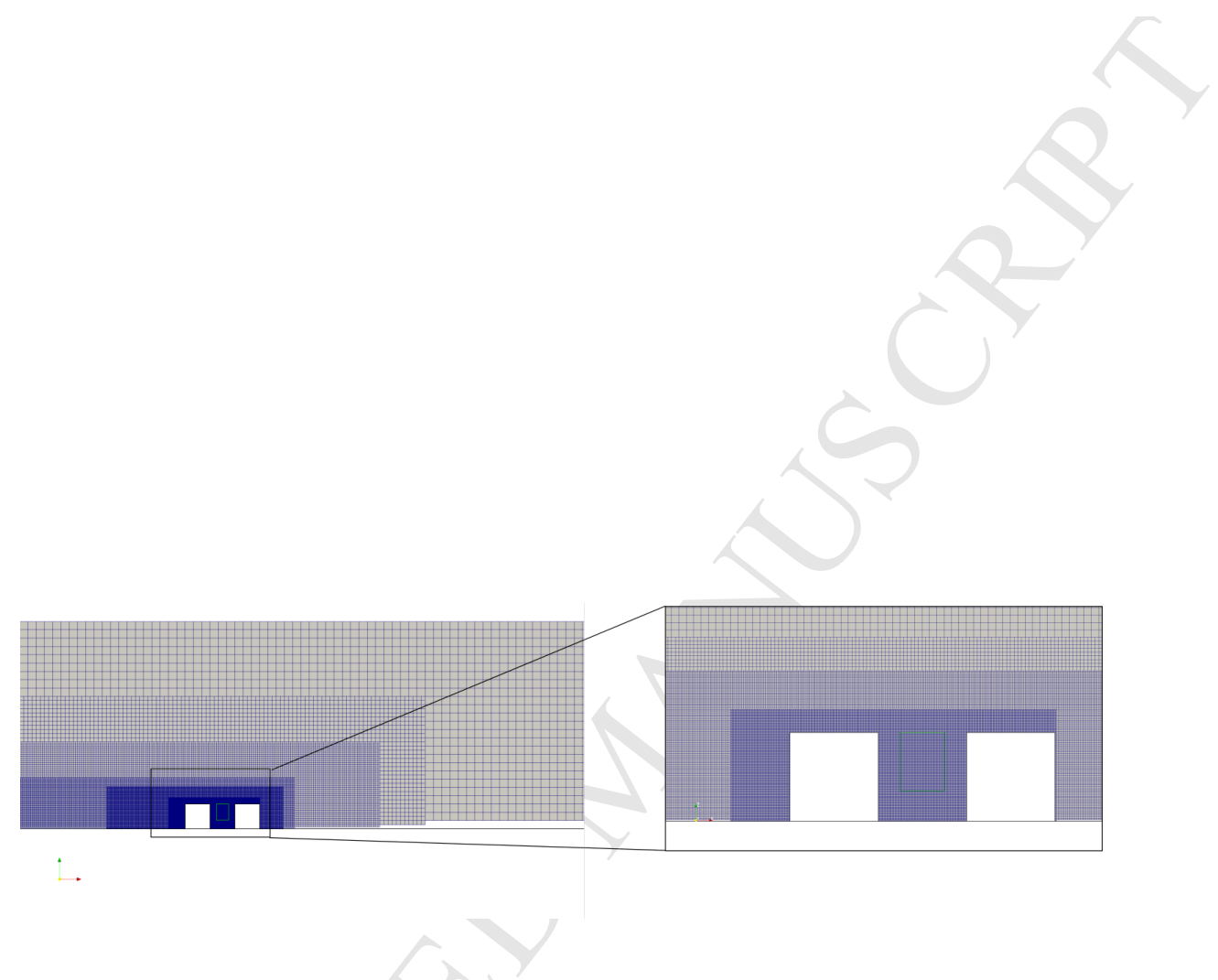

Figure 4: Computational mesh (5 refinement levels, $d x=\mathrm{H} / 96$ ) 

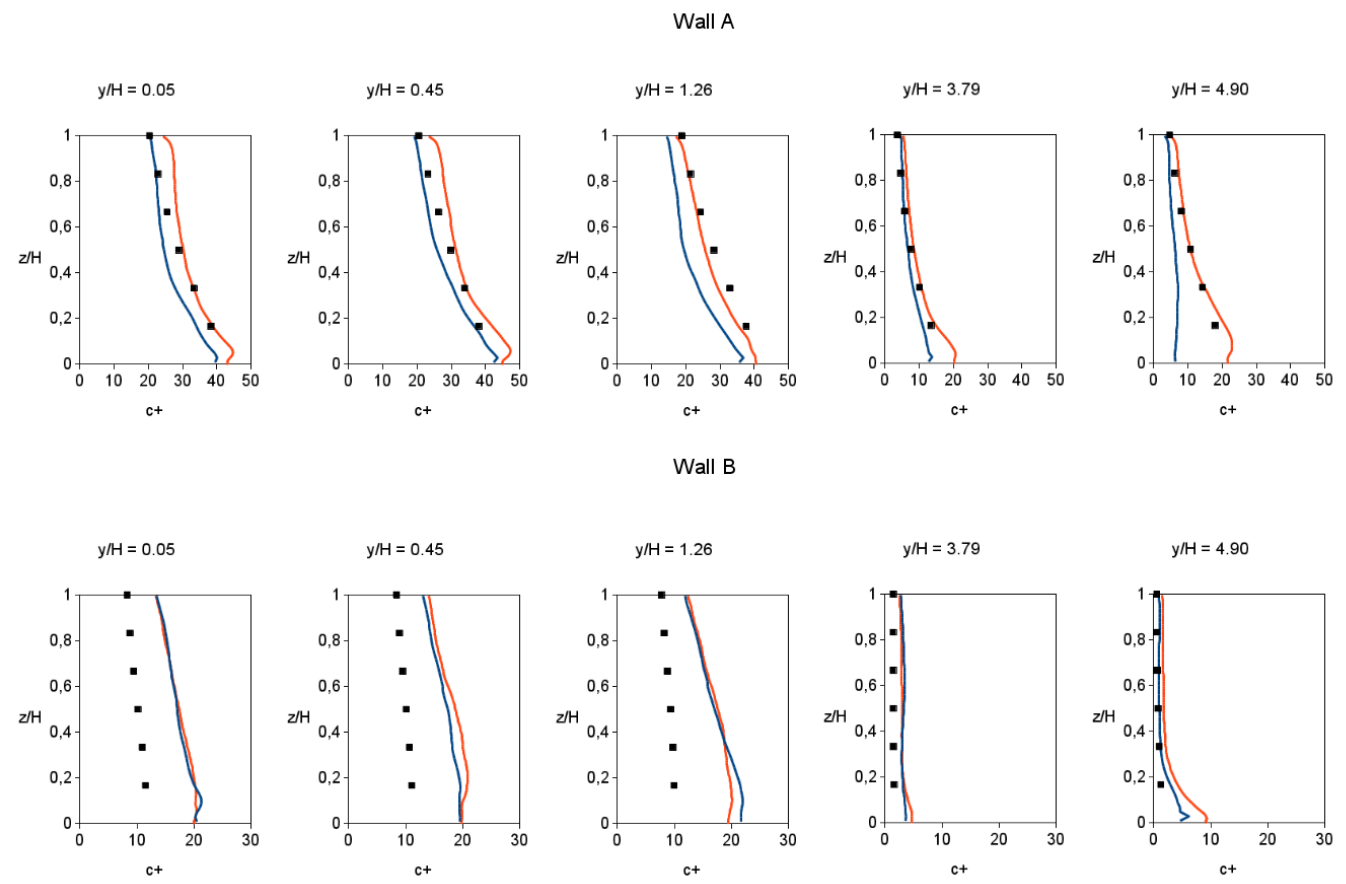

Figure 5: Comparisons between measured coarse (—) and fine (- $(-)$ grid 

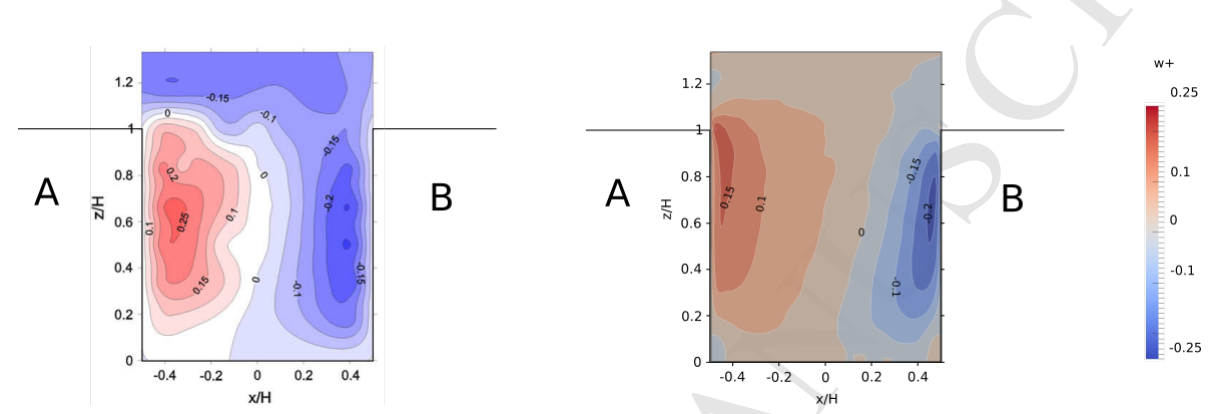

(a) $\lambda=0 \mathrm{~m}^{-1}$
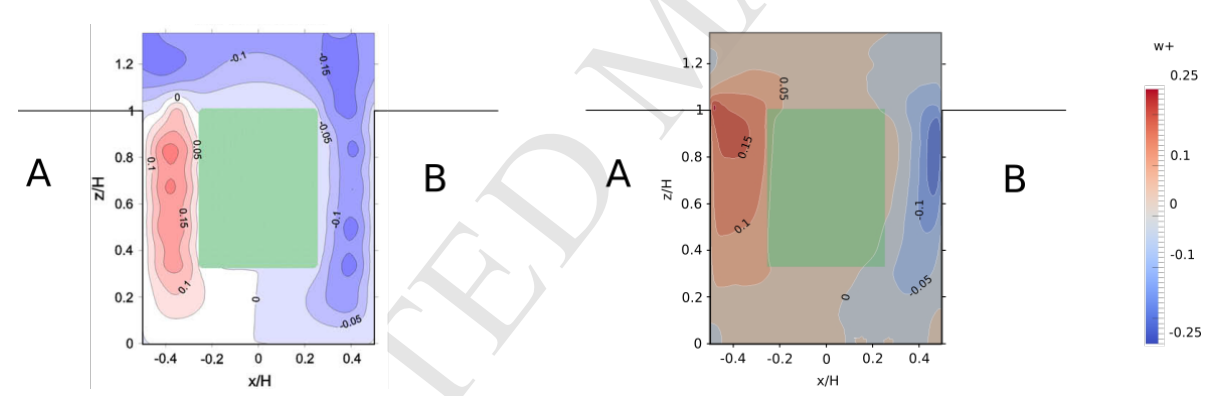

(b) $\lambda=250$ or $200 \mathrm{~m}^{-1}$

Figure 6: Comparison between measured (left - (Gromke et al., 2008)) and simulated (right) $w^{+}$contours in the plane $y / \mathrm{H}=0.5$ 


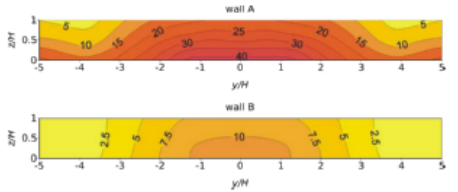

(a) Experiment $\lambda=0 m^{-1}$

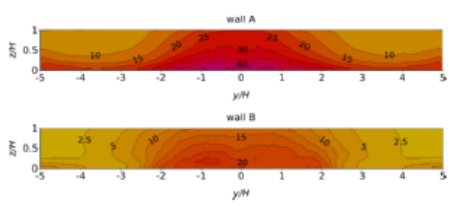

(d) Simulation $\lambda=0 m^{-1}$

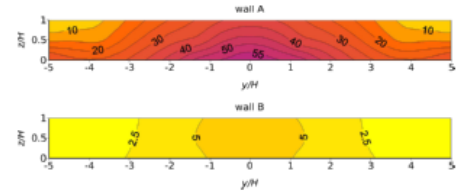

(b) Experiment $\lambda=80 \mathrm{~m}^{-1}$

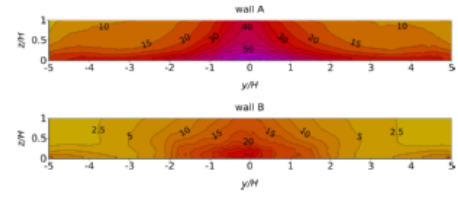

(e) Simulation $\lambda=80 \mathrm{~m}^{-1}$

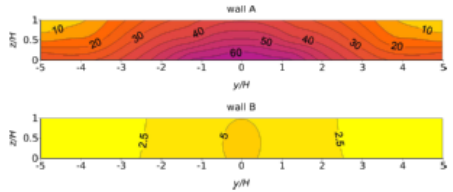

(c) Experiment $\lambda=200 \mathrm{~m}^{-1}$

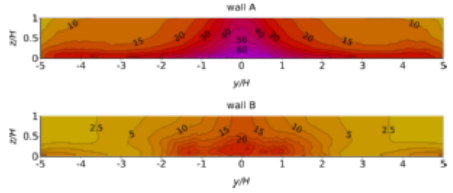

(f) Simulation $\lambda=200 \mathrm{~m}^{-1}$

Figure 7: $c^{+}$contours for $\lambda=0 \mathrm{~m}^{-1}, \lambda=80 \mathrm{~m}^{-1}$ and $\lambda=200 \mathrm{~m}^{-1}$, experimental Figure (C) $(\mathrm{KIT}, 2017)$ 
Wall A
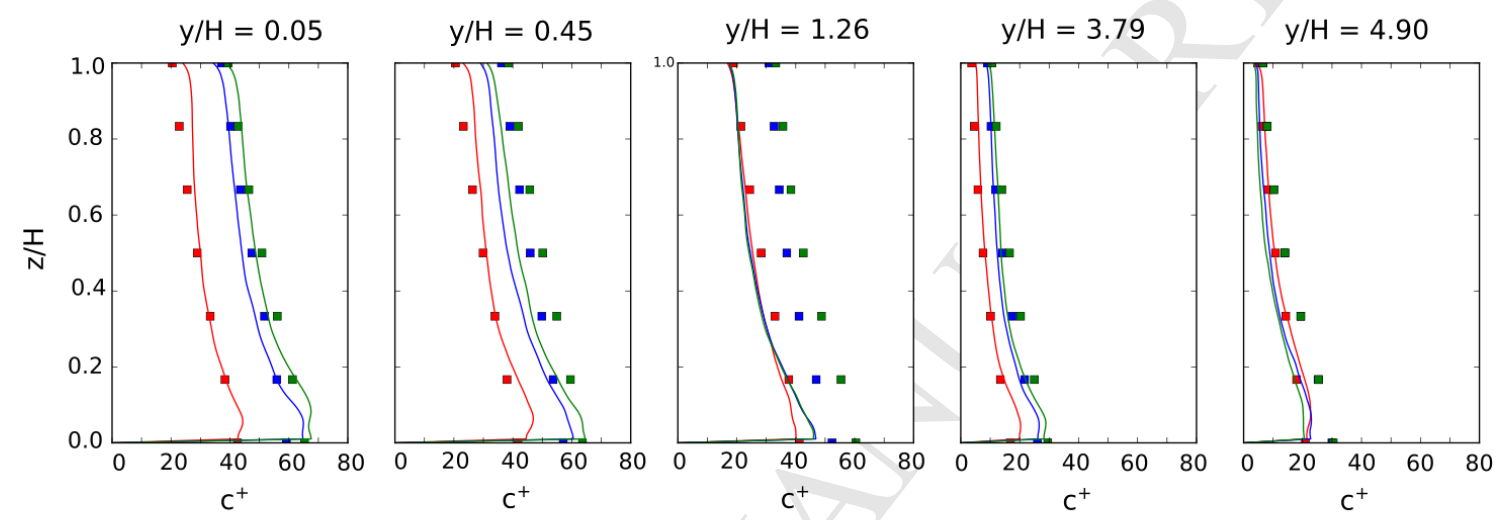

\section{Wall B}
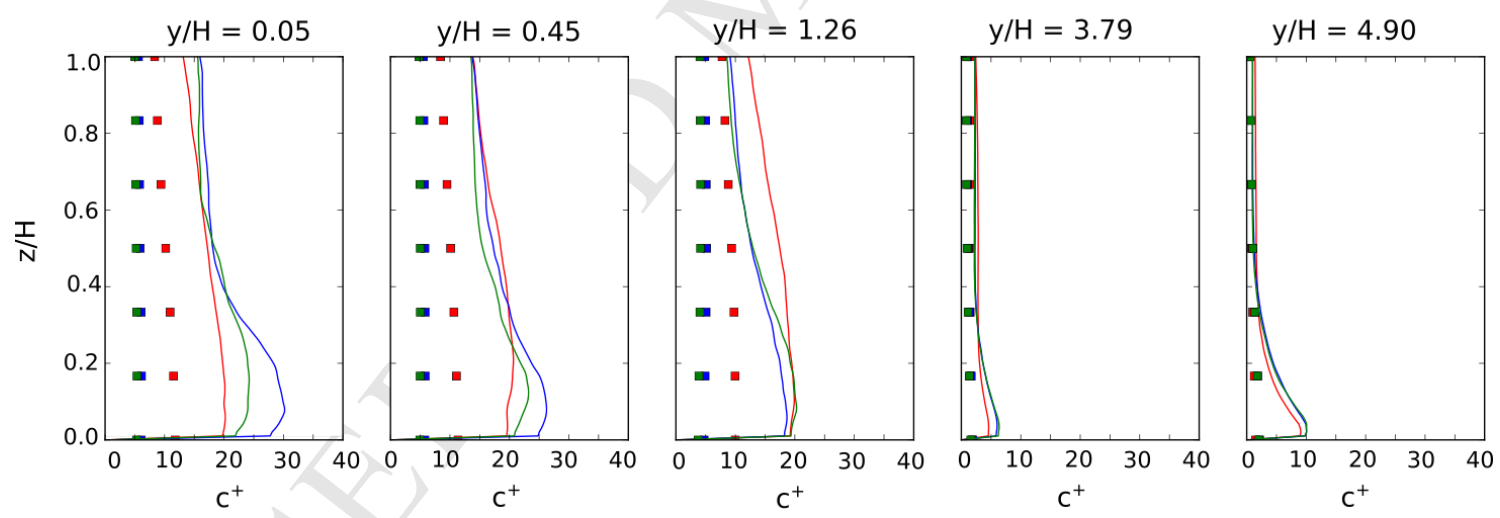

$$
\begin{array}{lll}
\text { - experiment, } \lambda=0 \mathrm{~m}^{-1} & \text { - experiment, } \lambda=80 \mathrm{~m}^{-1} & \text { - experiment, } \lambda=200 \mathrm{~m}^{-1} \\
\text { - simulation, } \lambda=0 \mathrm{~m}^{-1} & \text { - simulation, } \lambda=80 \mathrm{~m}^{-1} & \text { - simulation, } \lambda=200 \mathrm{~m}^{-1}
\end{array}
$$

Figure 8: Comparison between measured and simulated $c^{+}$vertical profiles 


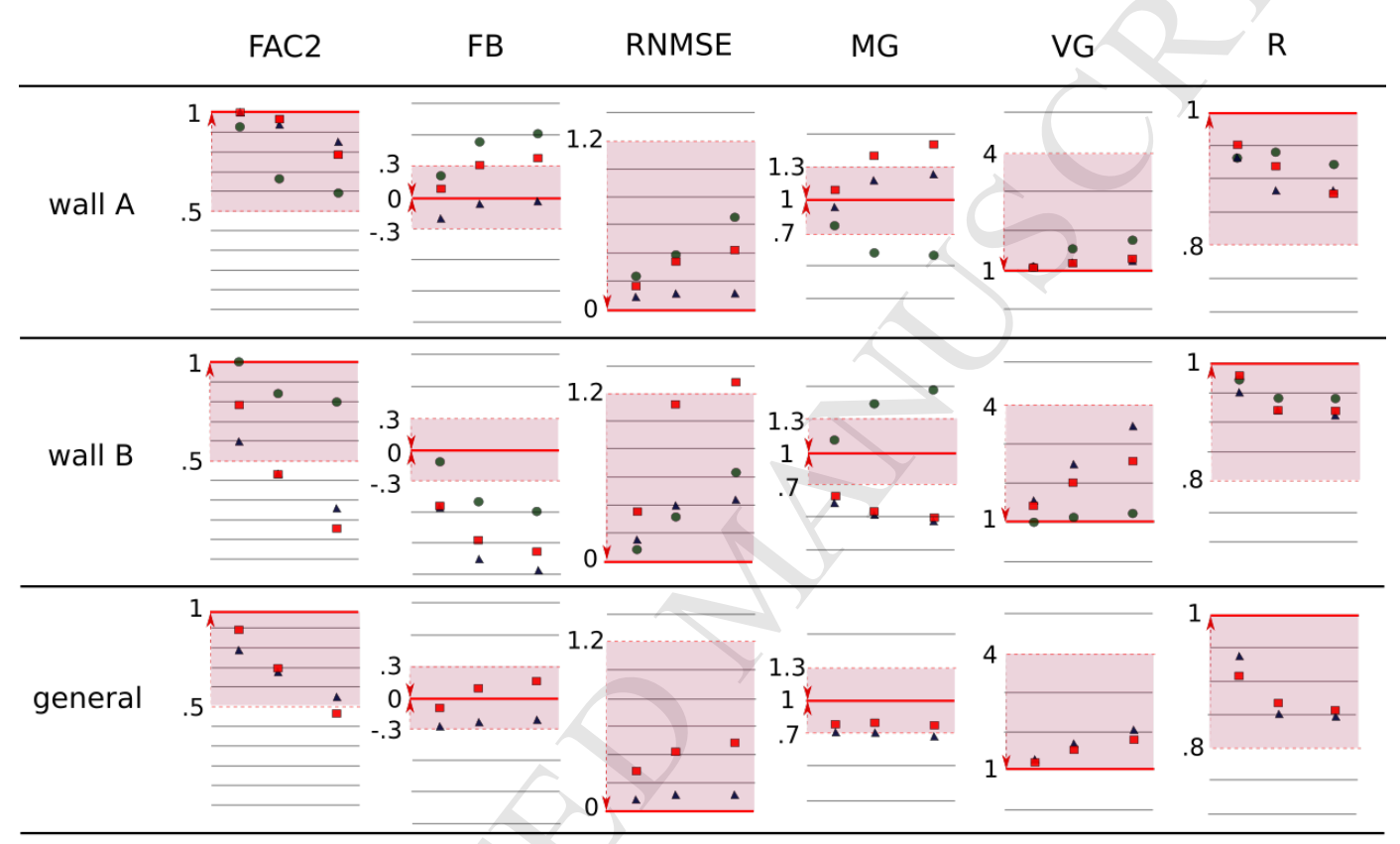

- ProLB (2018)

ـ Moonen et al. (2013)

- Kang et al. (2017)

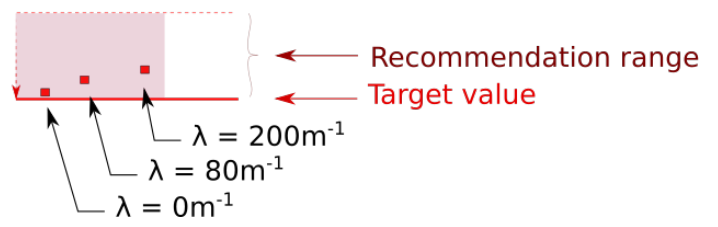

Figure 9: Comparison of quality metrics obtained using ProLB with results of Moonen et al. (2013) and Kang et al. (2017) 


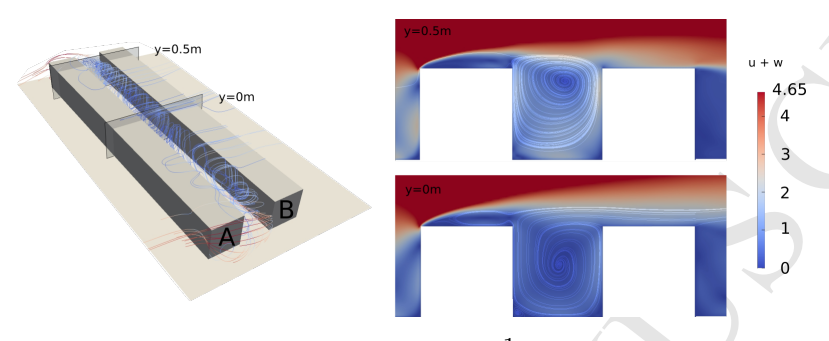

(a) $\lambda=0 \mathrm{~m}^{-1}$

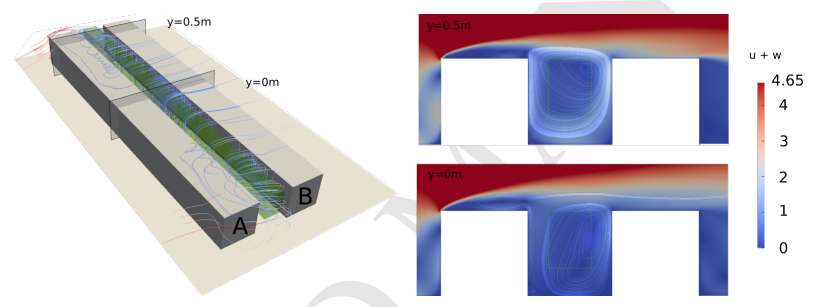

(b) $\lambda=80 \mathrm{~m}^{-1}$

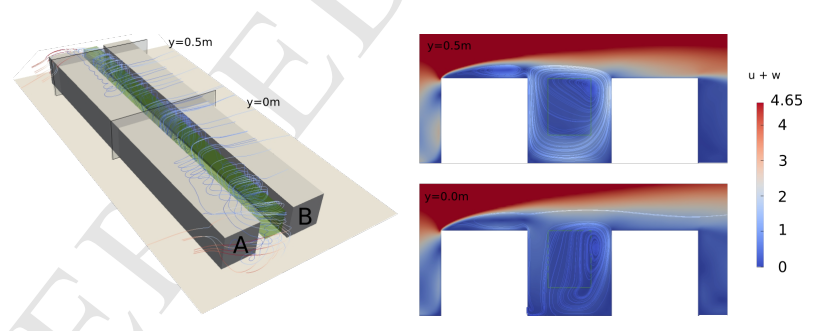

(c) $\lambda=200 \mathrm{~m}^{-1}$

Figure 10: Simulated mean velocity streamlines: 3D view (left) and in a 2D plane (right) 

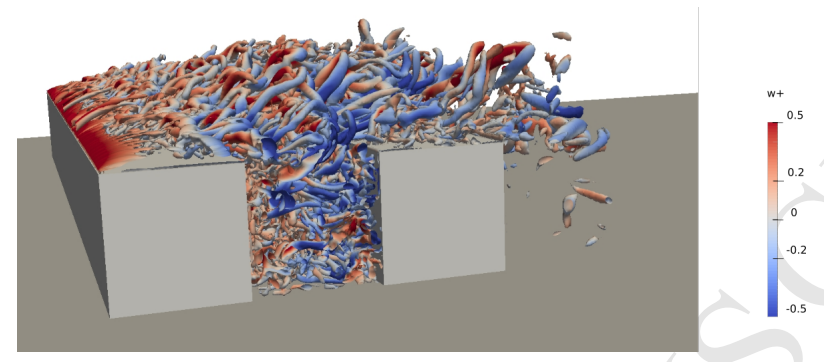

(a) $\lambda=0 \mathrm{~m}^{-1}$

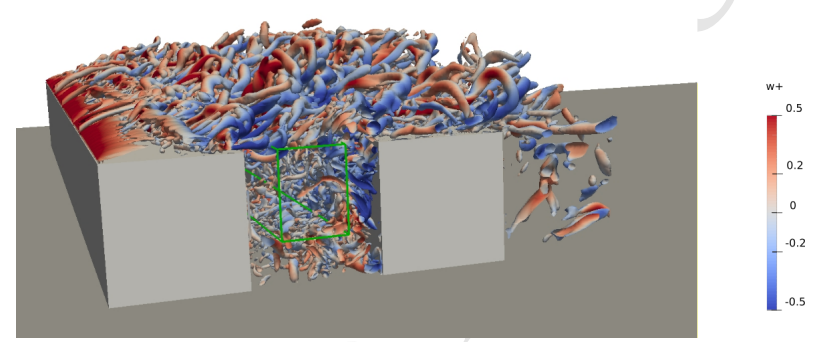

(b) $\lambda=80 \mathrm{~m}^{-1}$

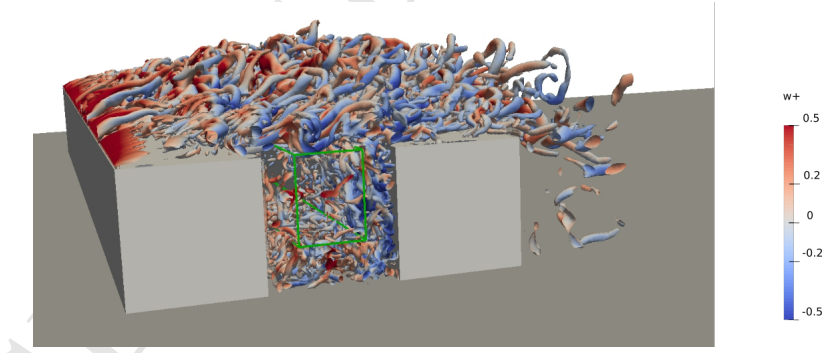

(c) $\lambda=200 \mathrm{~m}^{-1}$

Figure 11: $3 \mathrm{D}$ isocontours of the $\mathrm{Q}$ criterion $\left(Q=5 \times 10^{4}\right)$ colored by $w^{+}$, the facing section correspond to $y / H=-5$ 


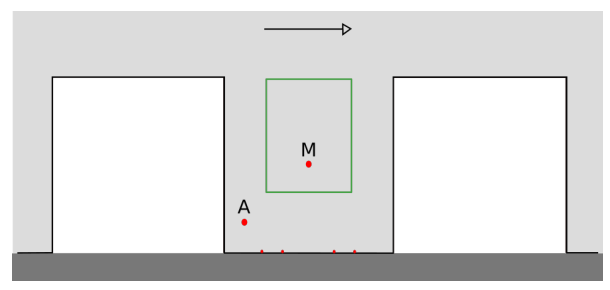

(a) Location of points A and M

Point A
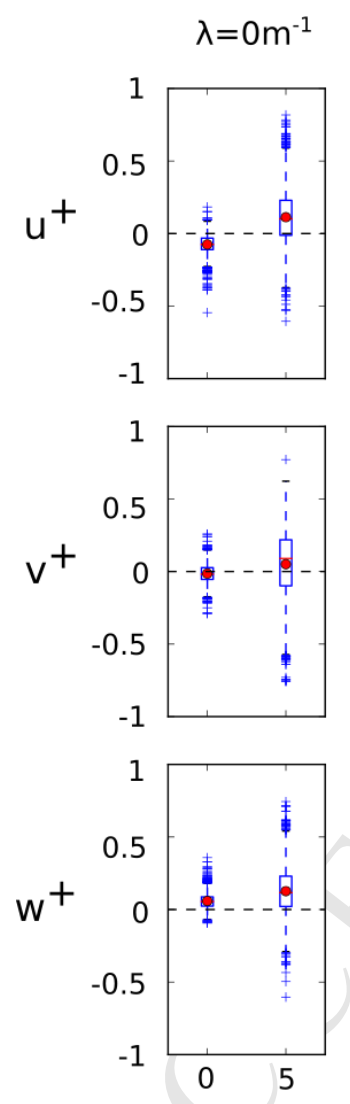
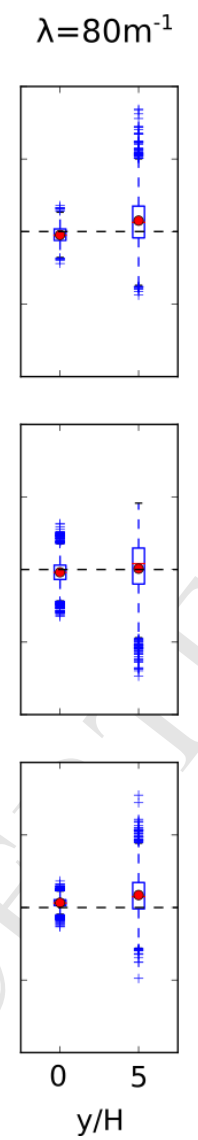
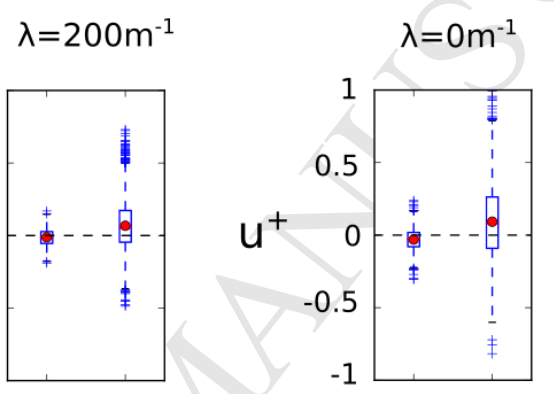

Point M
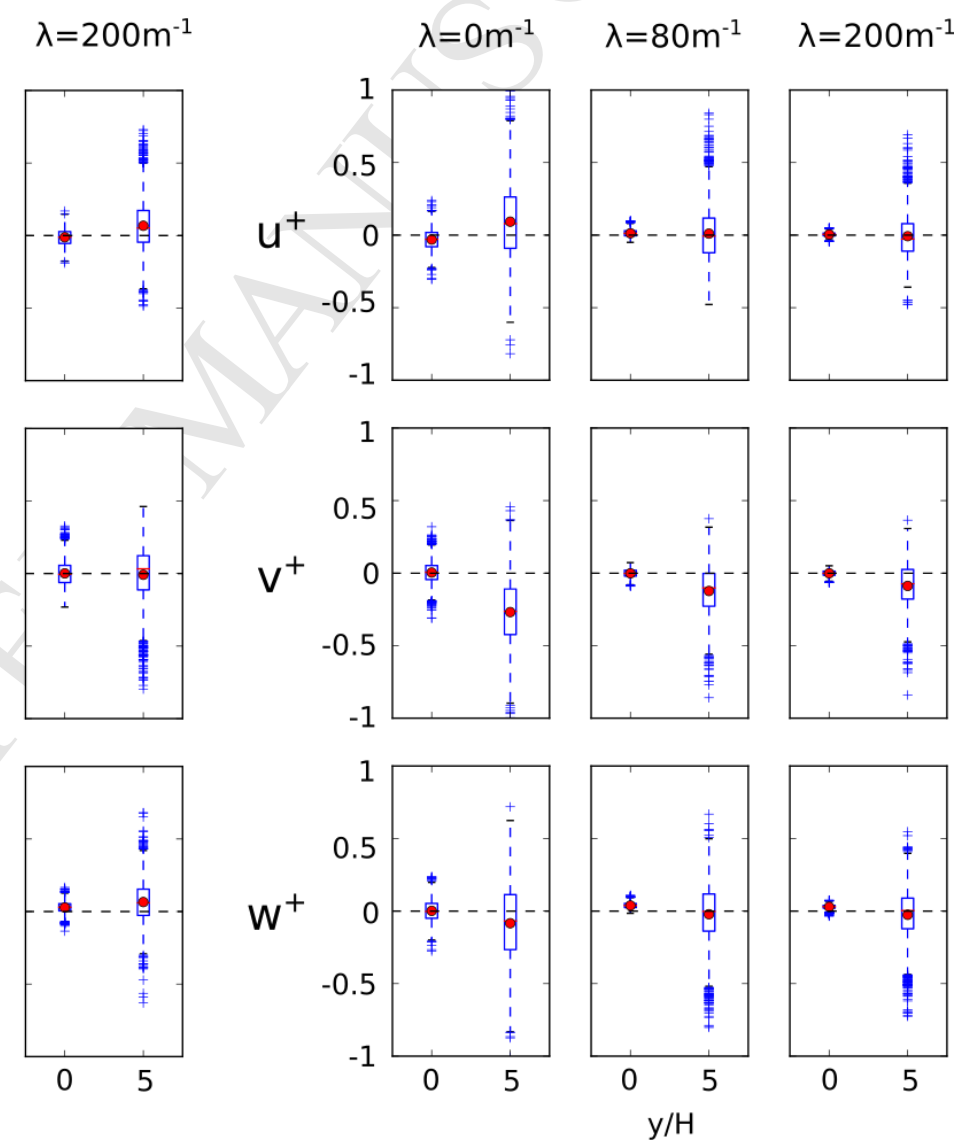

(b) Boxplot of $u^{+}, v^{+}, w^{+}$

Figure 12: Boxplot of instantaneous velocity components at points A and M 


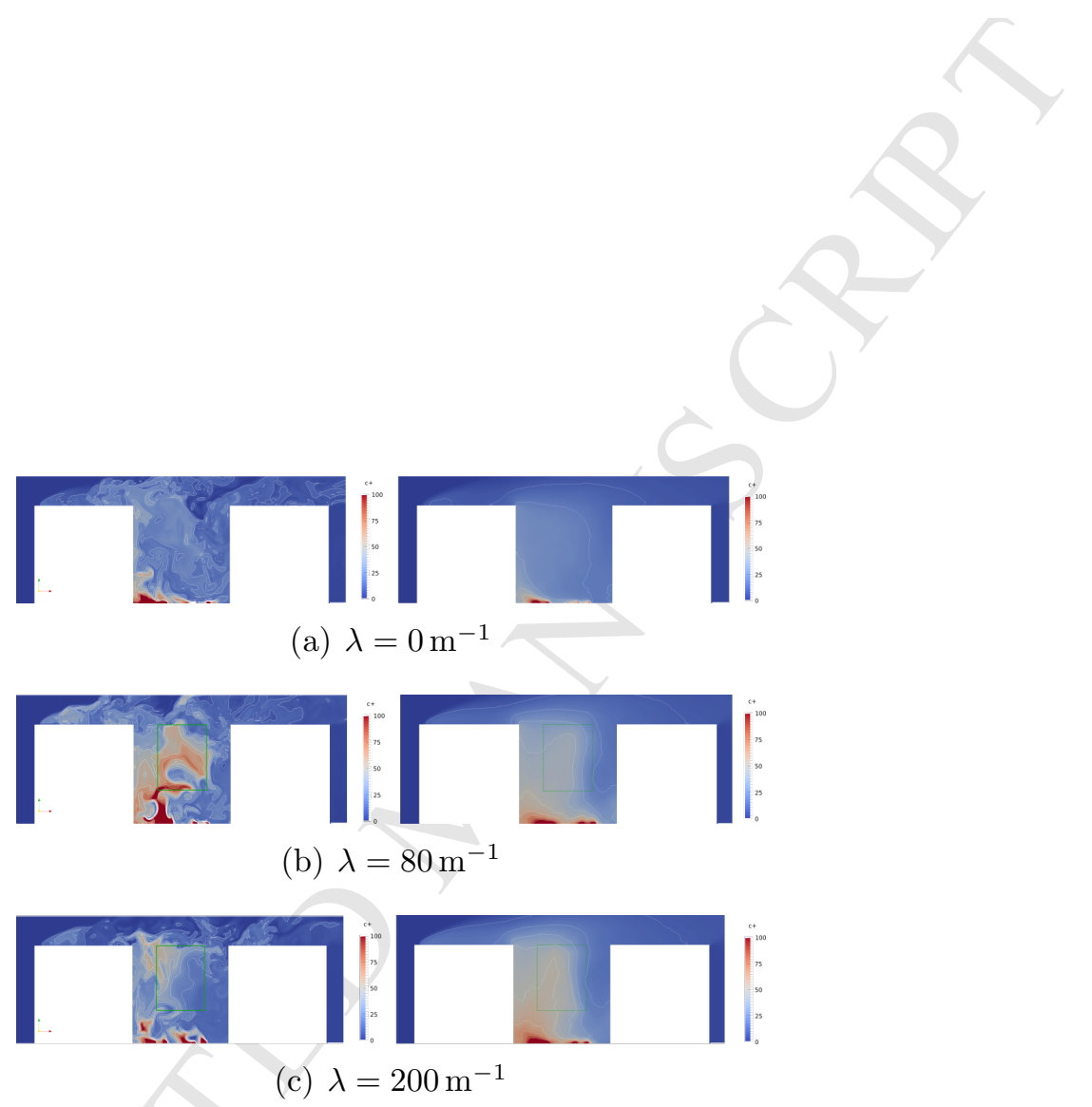

Figure 13: Simulated instantaneous (left) and mean (right) $c^{+}$fields in the $y / \mathrm{H}=0$ plane 
Point A

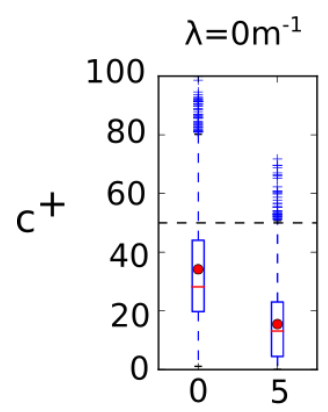

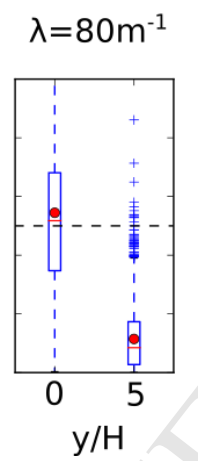
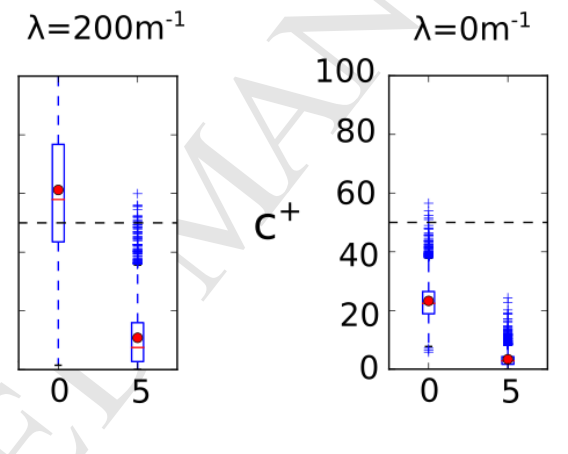

Point $M$

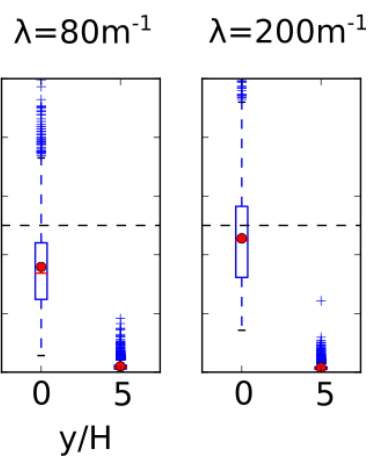

Figure 14: Boxplot of instantaneous concentration $\left(c^{+}\right)$ 
866

867

868

869

870

871

872

\section{List of Tables}

1 Overview of CFD studies dealing with the CODASC benchmark, $\mathrm{H} / \mathrm{W}=1$, trees and a wind perpendicular to the street canyon . . . . . . . . . . . . . . . 57

2 Statistical model performance indicators . . . . . . . . . 58

3 Statistical model performance indicators computed for the coarse and fine grid at both wall in the tree free configuration . . . . 59 


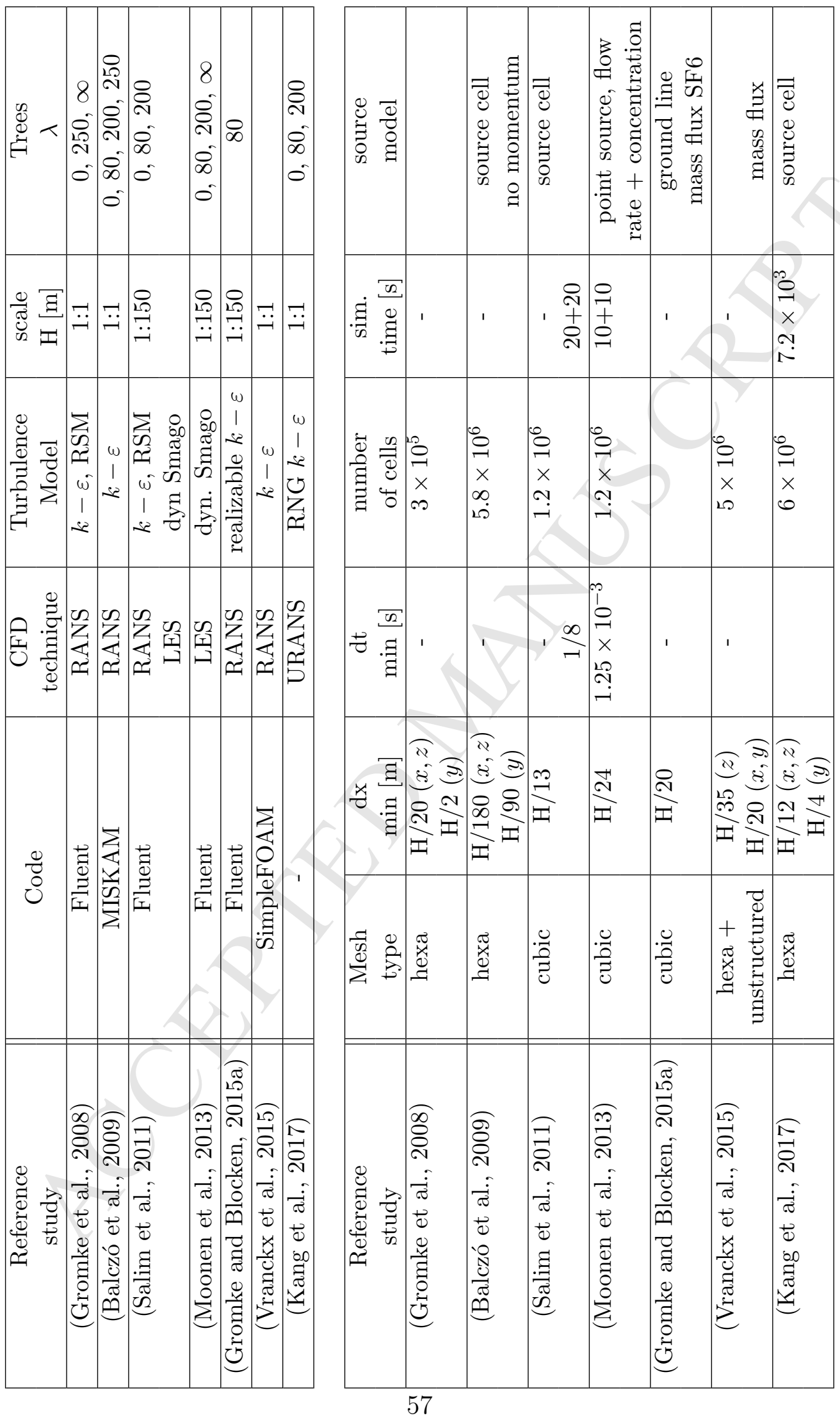

Table 1: Overview of CFD studies dealing with the CODASC benchmark, $\mathrm{H} / \mathrm{W}=1$, trees and a wind perpendicular to the street canyon 


\begin{tabular}{|c|c|c|c|c|c|c|}
\hline 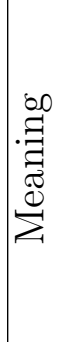 & 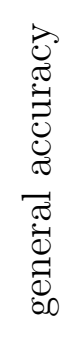 & 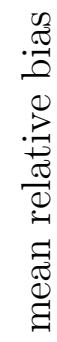 & 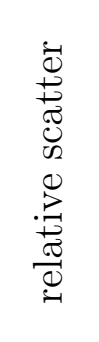 & 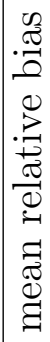 & 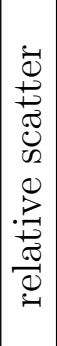 & 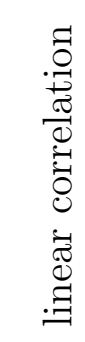 \\
\hline 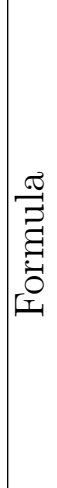 & 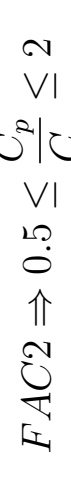 & 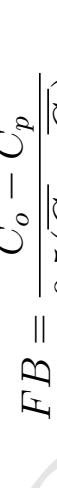 & $\left.\left.\underbrace{\left.\underbrace{2}\right|^{2} \mid}\right|^{2}\right|^{0}$ & 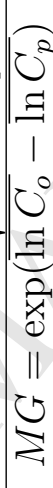 & 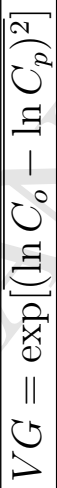 & 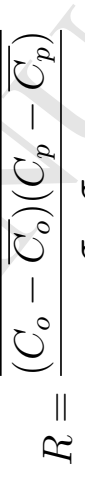 \\
\hline 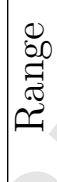 & $\begin{array}{l}20 \\
0 \\
\Lambda\end{array}$ & $\begin{array}{l}0 \\
0 \\
0 \\
0 \\
0\end{array}$ & $\stackrel{\sim}{\sim}$ & 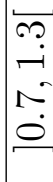 & $\begin{array}{l}F^{\prime} \\
V\end{array}$ & $\begin{array}{l}\infty \\
\dot{0} \\
\wedge\end{array}$ \\
\hline$\stackrel{\Xi}{\gtrless}$ & $\digamma$ & 0 & 0 & $\neg$ & -1 & $\neg$ \\
\hline 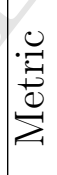 & 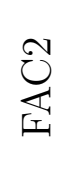 & $\stackrel{\rho}{I_{I}}$ & 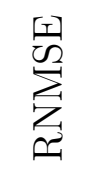 & $\stackrel{\square}{\Sigma}$ & $\stackrel{P}{P}$ & 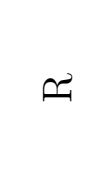 \\
\hline
\end{tabular}

Table 2: Statistical model performance indicators 


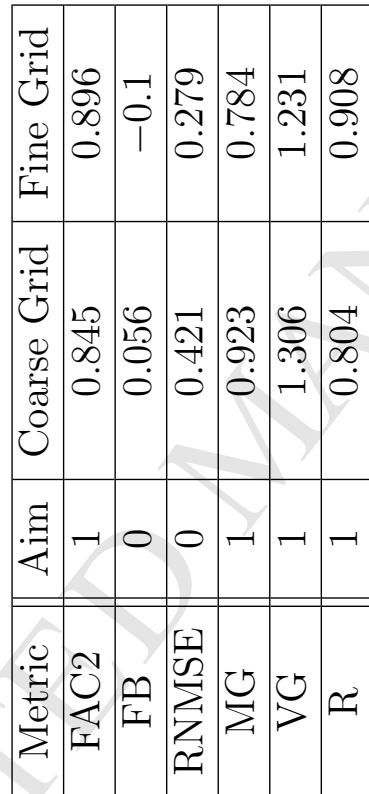

Table 3: Statistical model performance indicators computed for the coarse and fine grid at both wall in the tree free configuration 
Highlights:

- Pollutant dispersion in a street canyon using LES lattice Boltzmann method

- Rigorous model performance assessment with respect to wind tunnel measurements

- Analysis of the impact of trees on the fluctuating and time averaged velocity fields

- Analysis of the impact of trees on dynamic pollutant dispersion processes

- LBM-LES appears well suited for pollutant dispersion studies with tree plantings 